\title{
Ergodic Properties of the Quantum Ideal Gas in the Maxwell-Boltzmann Statistics
}

\author{
Marco Lenci * \\ Dipartimento di Matematica \\ Università di Bologna \\ 40127 Bologna \\ Italy
}

April 1996

\begin{abstract}
It is proved that the quantization of the Volkovyski-Sinai model of ideal gas (in the Maxwell-Boltzmann statistics) enjoys at the thermodynamical limit the property of quantum mixing in the following sense:

$\lim _{|t| \rightarrow \infty} \lim _{\substack{m, L \rightarrow \infty \\ m / L \rightarrow \rho}} \omega_{\beta, L}^{m}\left(e^{i H_{m} t / \hbar} A e^{-i H_{m} t / \hbar} B\right)=\lim _{\substack{m, L \rightarrow \infty \\ m / L \rightarrow \rho}} \omega_{\beta, L}^{m}(A) \cdot \lim _{\substack{m, L \rightarrow \infty \\ m / L \rightarrow \rho}} \omega_{\beta, L}^{m}(B)$.
\end{abstract}

Here $H_{m}$ is the Schrödinger operator of $m$ free particles moving on a circle of length $L ; A$ and $B$ are the Weyl quantization of two classical observables $a$ and $b ; \omega_{\beta, L}^{m}(A)$ is the corresponding quantum Gibbs state. Moreover one has

$$
\lim _{\substack{m, L \rightarrow \infty \\ m / L \rightarrow \rho}} \omega_{\beta, m}(A)=P_{\rho, \beta}(a)
$$

where $P_{\rho, \beta}(a)$ is the classical Gibbs measure.

The consequent notion of quantum ergodicity is also independently proven.

* On leave at: Mathematics Department, Princeton University, Princeton, NJ 08544, U.S.A. E-mail: marco@math.princeton.edu 


\section{Introduction.}

The purpose of the present paper is to analyze the quantum ergodic properties of the Volkovyski-Sinai model of ideal gas, quantized according to a Maxwell-Boltzmann statistics (i.e. all particles are distinguishable). This paper represents the companion paper of G-M where the same result is proved for a strongly analogous system, namely the infinite harmonic chain with suitable restrictions on the normal mode frequencies.

These two systems provide examples of kinematic quantum chaos. We borrow the expression kinematic chaos from the enlightening paper by [JL-P]. By that we mean trivial motion whose chaotic behavior is due to the randomness of the infinite-dimensional initial condition (see, besides [JL-P itself, the remark after Theorem 3.3). For both the ideal gas and the harmonic chain, this is a classical feature of the system, which works on the quantum level as well because of the exact Egorov Theorem (Lemma 4.1). The latter is a fundamental fact here, since it allows us to treat the quantum time evolution as the classical one.

A clear explanation of the motivations for the investigation of the quantum behavior of infinite systems is given in G-M], as well as further references. Here we just sketch what the immediate problems are, concerning the search for quantum chaos.

To fix the ideas, let $H$ be a self-adjoint operator acting on $L^{2}(\Omega), \Omega \in \mathbb{R}^{m}$, resulting from the quantization of some classical Hamiltonian function $\mathcal{H}$ over $\mathbb{R}^{m} \times \Omega$. Suppose, as it happens in all interesting cases, that $\sigma(H)$ is discrete. Consider two operators $A, B \in \mathcal{L}\left(L^{2}(\Omega)\right)$, which is regarded as our set of observables, and define $\Theta[t](A):=e^{i H t / \hbar} A e^{-i H t / \hbar}$, the Heisenberg evolution. All the physical experiments one can do on such a system imply a certain "measure" on the observables is used. In quantum mechanics such measures are states over the algebra of the operators. These quantum ensembles ( $\mathbb{R}]$, $\S 1.3)$ are typically described as

$$
\omega_{\varrho}(A)=\frac{\operatorname{Tr} A \varrho}{\operatorname{Tr} \varrho}
$$

with $\varrho$ a suitable trace-class operator. A suitable definition of mixing would be, then $(\mathrm{B}-\mathrm{R}], \mathrm{B}, \mathrm{G}-\mathrm{M})$

$$
\lim _{|t| \rightarrow \infty} \omega_{\varrho}(\Theta[t](A), B)=\omega_{\varrho}(A) \omega_{\varrho}(B), \quad \forall A, B \in \mathcal{L}\left(L^{2}(\Omega)\right) .
$$


It is easily realized, writing $\omega_{\varrho}$ by means of the matrix elements of the operators, w.r.t. the eigenbasis of $H$, that such a property can never be verified, for each underlying classical dynamics. The same is true for any reasonable definition of ergodicity, as Von Neumann's formula ( $[\overline{V N})$ shows (see $G-M$ for details). This is a consequence of the quasi-periodicity of the classical evolution - as long as we have finite degrees of freedom - and it is called "quantum suppression of classical chaos" ([JL-P-C], [JL-P]). Hence the idea of taking the number of degrees of freedom to infinity.

The system we consider is the quantization of the ideal gas in the formulation given in $[\mathbf{S}]$ : i.e. $m$ non-interacting particles moving freely on a circle of length $L$, when $m$ and $L$ are taken to infinity, subject to the finite density requirement $m / L \rightarrow \rho$.

For an outline of the "analytic approach" we will follow in studying the quantum infinite system in question, the reader is definitely referred to the introduction of the companion paper G-M, due to the similarities of the two works. There the consequences of such a study are also properly emphasized. In the next paragraph we just point out the structural differences, between the two models, which require non obvious modifications of the arguments of G-M valid for the harmonic chain. Specifically

(i) The other important classical mechanism which provides the unpredictability (mixing) of the time evolution here, besides the mentioned kinematic effect, is the symmetry of the observables under particle exchange. This corresponds to the physical fact that one is not able to distinguish between the particles in a gas. Actually, such a restriction on the observables also has the noticeable outcome to allow the interchange of the time average limit with the thermodynamical limit (see Theorem 3.3, Corollary 3.4, and relation (3.19)). The remark after Theorem 3.3 will contain more comments. On the quantum side, the simmetry of the observables would entail for a Bose-Einsten or a FermiDirac statistics. Nevertheless, we use Maxwell-Boltzmann for the sake of convenience: see remark 2 in $\oint 2.2$.

(ii) The phase space of any $m$-particle subsystem is $\mathbb{R}^{m} \times\left(L S^{1}\right)^{m}$, with $L S^{1}$ denoting henceforth the circle of radius $L$. In other words the phase space has a cylindrical structure. This has the effect of making necessary a Bloch decomposition of $L^{2}\left(\mathbb{R}^{m}\right)$, and consequently a direct fiber decomposition of operators, if we want to consider functions on 
phase space as symbols of operators on a one-to-one correspondence under quantization. Equivalently, $\mathbb{R}^{m} \times\left(L S^{1}\right)^{m}$ generates a cylindrical Heisenberg group (see [aB-G and $\delta 2.2$ ) and its only faithful unitary representation is given by a fibered $L^{2}$ space.

(iii) The coherent states we use are those adapted to the cylindrical phase space, as presented in $\S \mathrm{A} .1$ (refer also to $[\mathrm{P}]$, dB-G]).

(iv) The infinite-particle limit is a true thermodynamic limit, because here not only we have $m \rightarrow \infty$, but also $L \rightarrow \infty$ under the constraint of finite density $\frac{m}{L} \rightarrow \rho, \rho>0$.

(v) On the other hand in this model the classical dynamics is just free motion. This entails a simplification: the Weyl symbol of the quantum $m$-particle Schrödinger operator $H_{m}$ is obviously the classical Hamiltonian $\mathcal{H}_{m}=(1 / 2) \sum_{i=1}^{m} p_{i}^{2}$, namely $H_{m}=\operatorname{Op}\left(\mathcal{H}_{m}\right)$. Then the Weyl symbol of the (unnormalized here) quantum Gibbs measure is

$$
e^{-\beta H_{m}}=\operatorname{Op}\left(e^{-\beta \mathcal{H}_{m}}\right),
$$

where, here and above, $\mathrm{Op}:=\mathrm{Op}^{W}$ denotes the operation of Weyl quantization of a symbol, whose definition in the present context is recalled in $\$ 2.2$ below.

Now, as it will be explained in sharper detail in $\$ 3.1$, consider the normalized quantum Gibbs state at inverse temperature $\beta$ (for a system of $m$ free particles in $L S^{1}$ ), namely the functional

$$
\omega_{\beta, L}^{m}(A):=\frac{\operatorname{Tr} A e^{-\beta H_{m}}}{\operatorname{Tr} e^{-\beta H_{m}}},
$$

defined, for instance, over the class of bounded operators $A$ which are " $L$ periodic in the coordinate variable" (refer to $\$ 2.2$ ). The application $\mathrm{Tr}$ is practically the trace over $L^{2}\left(\mathbb{R}^{m}\right)$ (see $\$ 3.1$ for details). Then the main result of the present work, in analogy with G-M, can be stated as

$$
\lim _{|t| \rightarrow \infty} \lim _{\substack{m, L \rightarrow \infty \\ m / L \rightarrow \rho}} \omega_{\beta, L}^{m}\left(\Theta^{m}[t](A) B\right)=\lim _{\substack{m, L \rightarrow \infty \\ m / L \rightarrow \rho}} \omega_{\beta, L}^{m}(A) \cdot \lim _{\substack{m, L \rightarrow \infty \\ m / L \rightarrow \rho}} \omega_{\beta, L}^{m}(B) .
$$

(see also Theorem 3.2 and (3.18) ). Here $A$ and $B$ are actually dependent on $L$ and $m$ and represent the operators quantizing two classical observables, $a$ 
and $b$, over the Hilbert spaces for $m$ particles in a $L$-circle (square integrable Bloch functions). Also $\Theta^{m}[t](A):=e^{i H_{m} t / \hbar} A e^{-i H_{m} t / \hbar}$. Moreover

$$
\lim _{\substack{m, L \rightarrow \infty \\ m / L \rightarrow \rho}} \omega_{\beta, m}(A)=P_{\rho, \beta}(a)
$$

Formula (1.4) is the quantum mixing property and induces a consistent formulation of the quantum ergodicity: for instance

$$
\lim _{T \rightarrow \infty} \lim _{\substack{m, L \rightarrow \infty \\ m / L \rightarrow \rho}} \omega_{\beta, L}^{m}\left(\left(\Xi^{m}[T](A)\right)^{2}\right)=\lim _{\substack{m, L \rightarrow \infty \\ m / L \rightarrow \rho}}\left(\omega_{\beta, L}^{m}(A)\right)^{2}
$$

if $\Xi^{m}[T](A)=(1 / 2 T) \int_{-T}^{T} \Theta^{m}[t](A) d t$.

The present model of quantum ideal gas is structurally different from the free Bose gas or the free Fermi gas in the grandcanonical ensemble, discussed e.g. in $[\mathrm{B}-\mathrm{R}], \S 5.2$. The arguments $(\mathbb{B}-\mathrm{R}],[\mathrm{B}]$ ) yielding the mixing property with respect to the KMS states through the asymptotic abelianess of the CCR (or CAR) automorphisms generated by free dynamics do not apply in this context.

The paper is organized as follows: in the next section we briefly recall the ideal gas model and construct its quantization; in $\$ 3$ we state the results, whose proofs are given in $\S$. Finally, in the appendix we recall the construction of the coherent states on the cylinder (obtained e.g. in $[\mathrm{dB}-\mathrm{G}$ ) with some additional details; we collect two technical lemmas; and we exploit the properties of the convolution over $L S^{1}$, as $L \rightarrow \infty$, which are crucially used in the proofs.

\section{The Classical Ideal Gas and Its Quantiza- tion.}

\subsection{The Volkovyski-Sinai model.}

To make the exposition self-contained, a brief reminder is here given of the Volkovyski-Sinai model of ideal gas. The reader is referred to [S], Lecture 8, for details. 
Consider a system of $m$ free particles of unit mass constrained to move on a circle of length $L$. Its Hamiltonian function is

$$
\mathcal{H}_{m}(p, q)=\frac{1}{2} \sum_{j=1}^{m} p_{j}^{2}=p^{2} / 2
$$

defined on the phase space $\Lambda_{L}^{m}:=\mathbb{R}^{m} \times\left(L S^{1}\right)^{m}$ which, with a view to the limiting case $L \rightarrow \infty$ to be considered below, will be identified with $\mathbb{R}^{m} \times T_{L}^{m}, T_{L}^{m}:=[-L / 2, L / 2)^{m}$. The physical intuition is to stretch the circle $L S^{1}$ more and more towards a straight line, namely $\mathbb{R}$.

On $\Lambda_{L}^{m}$ the motion is given by the flow map: $\phi_{L}^{m}[t](p, q):=(p, q+p t)$. The dynamical system is completely integrable and $\Lambda_{L}^{m}$ is decomposed in a noncountable family of invariant tori: all motions are quasi-periodic. Introducing any "reasonable" measure on $\Lambda_{L}^{m}$ (in [S] the microcanonical measure is used; for reasons which will become clear when quantizing, the natural measure to introduce here is the canonical one) the system is of course not even ergodic.

But a suitable thermodynamical limit of it might be. The construction of the infinite dynamical system is based on the idea that the particles should be "unlabeled" the particles. More precisely, the phase space for the infinite system is defined as follows:

$$
\Lambda_{\infty}:=\{(p, q) ; q \text { countable subset of } \mathbb{R}, p: q \rightarrow \mathbb{R}\}
$$

with the interpretation that $q=\left\{x_{1}, x_{2}, \ldots, x_{j}, \ldots\right\}$ contains the positions of the particles, now undistinguishable, and $p$ is a function such that $p\left(x_{j}\right)$ gives the velocity of the particle located at $x_{j}$. It may occur that more than one particle - say $n$ - are located at $x_{j}$ : in this case, with an abuse of notation with respect to definition (2.2), $p\left(x_{j}\right)$ is an $n$-tuple of velocities. The flow $\phi_{\infty}[t]: \Lambda_{\infty} \rightarrow \Lambda_{\infty}$ is defined accordingly: $\phi_{\infty}[t](p, q)=\left(p^{\prime}, q^{\prime}\right)$, where $q^{\prime}:=\{x+p(x) t ; x \in q\}$ and $p^{\prime}: q^{\prime} \rightarrow \mathbb{R}$ is $p^{\prime}(x+p(x) t):=p(x)$.

What we have introduced is the natural limiting object of the spaces $\Lambda_{L}^{m} / \mathbb{S}^{m}, \mathbb{S}^{m}$ being the group of permutations of $m$ coordinates and momenta. Such spaces are expressly defined in [S] in a way completely analogous to (2.2), i.e. as the collection of all couples $(p, q)$ with $q \subset L S^{1}, \# q \leq m$ and $p: q \rightarrow \mathbb{R}$ having $m$ values, in the sense specified above. They can be regarded as belonging to $\Lambda_{\infty}$, since $q \subset L S^{1} \simeq[-L / 2, L / 2)$. This motivates the above identification. 
This remark enables us to consider functions defined on $\Lambda_{\infty}$ as having a natural restriction on $\Lambda_{L}^{m} / \mathbb{S}^{m}$. And a function $f$ on $\Lambda_{L}^{m} / \mathbb{S}^{m}$ is simply a totally symmetric function on $\Lambda_{L}^{m}$, namely $f \circ \Pi_{L}^{m}$, where $\Pi_{L}^{m}$ is the natural immersion $\Lambda_{L}^{m} \rightarrow \Lambda_{\infty}$ :

$$
\Pi_{L}^{m}\left(p_{1}, \ldots, p_{m}, q_{1}, \ldots, q_{m}\right):=\left(p: q \rightarrow \mathbb{R}, q:=\left\{q_{1}, \ldots, q_{m}\right\}\right),
$$

$p$ taking values $p\left(q_{1}\right)=p_{1}, \ldots, p\left(q_{m}\right)=p_{m}$. It will be useful in the remainder to notice that

$$
\phi_{\infty}[t] \circ \Pi_{L}^{m}=\Pi_{L}^{m} \circ \phi_{L}^{m}[t]
$$

To complete the definition of our infinite dynamical system, we need to specify the measurable functions, i.e. to fix a $\sigma$-algebra $\mathcal{A}$ and - after that - a probability measure on it. The answer in $\mathrm{V}-\mathrm{S}$ is $\mathcal{A}:=\sigma(\gamma(\Delta))_{\Delta \in \mathcal{B}(\mathbb{R})}$. Here $\Delta$ runs among the Borel sets in $\mathbb{R}$ and $\gamma(\Delta)$ is the $\sigma$-algebra of all subsets of $\Lambda_{\infty}$ depending only on the positions and momenta of the unlabeled particles in $\Delta$. 円 For the sake of simplicity, we just restrict ourselves to real functions.

Examples of measurable functions are: $f_{\Delta}(p, q):=\#(q \cap \Delta)$, the number of particles of the configuration $q$ located in $\Delta$; or $g_{\Delta}(p, q):=\sum_{x \in \Delta} p(x)$, the total momentum of the particles in $\Delta$.

We endow $\mathcal{A}$ with the measure $P_{\rho, \beta}$ defined by the following properties:

1. The distribution of particles in the configuration space is Poissonian with parameter $\rho$; that is

$$
P_{\rho, \beta}\left(\left\{(p, q) ; f_{\Delta}(p, q)=n\right\}\right)=e^{-\rho|\Delta|} \frac{(\rho|\Delta|)^{n}}{n !} ;
$$

for every $\Delta \in \mathcal{B}(\mathbb{R})$. This implies that the distributions over two disjoint Borel sets $\Delta_{1}$ and $\Delta_{2}$ are independent.

2. The momenta are independent centered Gaussian variables with variance $1 / \beta$. This means that, fixed $A \in \mathcal{B}\left(\mathbb{R}^{n}\right)$ and a vector $\left(x_{1}, \ldots, x_{n}\right) \in$ $\mathbb{R}^{n}$, we have

$$
P_{\rho, \beta}\left(\left\{\left(p\left(x_{1}\right), \ldots, p\left(x_{n}\right)\right) \in A\right\} \mid\left\{\left\{x_{1}, \ldots, x_{n}\right\} \subset q\right\}\right)=\int_{A}\left[e^{-\beta p^{2} / 2} d p\right]_{N}
$$

\footnotetext{
${ }^{1}$ More precisely, $\gamma(\Delta)$ is the $\sigma$-algebra for which the measurable functions are all functions $f$ on $\Lambda_{\infty}$ depending only on values taken by the particles in $\Delta$ and measurable when viewed as functions on $\Lambda_{L}^{m}: f \circ \Pi_{L}^{m}$.
} 
$[\cdots]_{N}$ being the normalized measure. This is a Maxwell distribution with inverse temperature $\beta$.

This measure has been chosen intentionally as the limit of the canonical measures over the phase spaces of finite numbers of particles.

Proposition 2.1 Let a measurable in $\mathcal{A}$. At the thermodynamical limit, i.e. $m, L \rightarrow \infty ; m / L \rightarrow \rho$,

$$
\int_{\Lambda_{L}^{m}}\left(a \circ \Pi_{L}^{m}\right)(p, q)\left[e^{-\beta p^{2} / 2} d p d q\right]_{N} \longrightarrow P_{\rho, \beta}(a)
$$

As regards the proof, the statement concerning the distribution of the positions is clearly explained in [S], while (2.6) trivially holds since the distributions over the momentum spaces for a finite number of particles are given as Maxwellian with inverse temperature $\beta$.

We conclude this section formulating the key

Theorem 2.2 V-S The measure $P_{\rho, \beta}$ is invariant under $\phi_{\infty}[t]$ and the $d y$ namical system $\left(\Lambda_{\infty}, \mathcal{A}, P_{\rho, \beta}, \phi_{\infty}[t]\right)$ is a $K$-flow.

It may be worth noticing here that the infinite dynamical system just recalled has a nice abstract construction, which is described in G-L-A (and shortly in [B], Example 2.34). It is called the Poisson system constructed for a onedimensional free particle with a Maxwell velocity distribution. Its ergodic properties follow via a general technique, namely the Bernoulli construction. This view-point shows clearly that $\mathcal{A}$ is generated by the following sets:

$$
B_{\Delta, \Gamma}^{(n)}:=\left\{(p, q) \in \Lambda_{\infty} \mid \#\{x \in q \mid x \in \Delta, p(x) \in \Gamma\}=n\right\}
$$

where $\Delta, \Gamma \in \mathcal{B}(\mathbb{R})$. Such a remark will we useful while proving the statements.

Nonetheless the approach we have chosen has the advantage of constructing the infinite-particle dynamical system through a thermodynamical limit (see above, and in particular Proposition 2.1). This fact will be crucial throughout this paper. 


\subsection{The quantization}

The Hilbert spaces associated to a quantum system of $m$ particles on a circle of length $L$ are denoted by $L_{(k)}^{2}\left(T_{L}^{m}\right), k \in[0,1 / L)^{m}$. Each of these is defined as

$$
L_{(k)}^{2}\left(T_{L}^{m}\right):=\left\{f \text { on }\left.\mathbb{R}^{m}\left|\forall j \in \mathbb{Z}^{m}, f(q+L j)=e^{2 \pi i L k \cdot j} f(q), \int_{T_{L}^{m}}\right| f\right|^{2}<\infty\right\},
$$

that is the space of the Bloch functions of parameter $k$ which are squareintegrable on a given fundamental domain. Concerning this definition, we remark:

1. The above family of spaces is the familiar one for Schrödinger operators with periodic potential (see [R-S], §XIII.16). They have to be simultaneously considered for all values of $k \in[0,1 / L)^{m}$, otherwise the quantization application is not well defined since the Schrödinger representation of the Heisenberg group is not faithful: e.g., in one dimension, if we selected only $k=0$, then $T(L, 0)=T(0,0)=\mathbf{1}$ (see below).

As a matter of fact, the whole Hilbert space $L^{2}\left(\mathbb{R}^{m}\right)$ is recovered through the standard direct integral formula $(\mathbb{R - S})$

$$
\int_{[0,1 / L)^{m}}^{\oplus} L_{(k)}^{2}\left(T_{L}^{m}\right) d k \simeq L^{2}\left(\mathbb{R}^{m}\right) .
$$

More details are given in $\S$ A.1 and in $\mathrm{dB}-\mathrm{G}$. A basis for $L_{(k)}^{2}\left(T_{L}^{m}\right)$ is obviously $e_{\alpha}^{(k)}:=L^{-m / 2} e^{2 \pi i(\alpha+k) \cdot x}$, with $\alpha \in(\mathbb{Z} / L)^{m}$.

2. The choice of the Hilbert spaces in (2.8) corresponds to a MaxwellBoltzmann statistics, since we ask for no wave function symmetry with respect to particle permutations. One could also think of quantizing this system according to a Bose-Einstein or a Fermi-Dirac statistics. To give a physical explanation, we are considering particles which are in principle enumerable but our observables do not see this enumeration. This approximation makes sense in the semiclassical realm. \&

\footnotetext{
${ }^{2}$ On the other hand, we stress that this paper does not involve any kind of semiclassical limit.
} 
A further step towards the definition of the quantization application is the introduction of the Fourier transform and antitransform in $\Lambda_{L}^{m}$. We define first the dual of the phase space: $\left(\Lambda_{L}^{m}\right)^{*}:=\mathbb{R}^{m} \times\left(T_{L}^{m}\right)^{*}$ where $\left(T_{L}^{m}\right)^{*}:=$ $(\mathbb{Z} / L)^{m}$. Now, if $b \in \mathcal{S}\left(\Lambda_{L}^{m}\right)$, that is the Schwartz class of functions in $\Lambda_{L}^{m}$, then for $(\eta, \xi) \in\left(\Lambda_{L}^{m}\right)^{*}$, the Fourier transform of $b$ is

$$
\widehat{b}(\eta, \xi):=\int_{\mathbb{R}^{m}} \int_{T_{L}^{m}} b(p, q) e^{-2 \pi i(\eta \cdot p+\xi \cdot q)} d q d p .
$$

Correspondingly, the antitransformation is given by

$$
b(p, q):=\frac{1}{L^{m}} \sum_{\xi \in\left(T_{L}^{m}\right)^{*}} \int_{\mathbb{R}^{m}} \widehat{b}(\eta, \xi) e^{2 \pi i(p \cdot \eta+q \cdot \xi)} d \eta .
$$

The Heisenberg group to be considered in this situation is the naturally induced cylinder subgroup of the Heisenberg group on $\mathbb{R}^{2 m} \times \mathbb{R}$, namely $\left(\Lambda_{L}^{m}\right)^{*} \times \mathbb{R}$ endowed with the product law

$$
(\eta, \xi, \tau)\left(\eta^{\prime}, \xi^{\prime}, \tau^{\prime}\right)=\left(\eta+\eta, \xi+\xi^{\prime}, \tau+\tau^{\prime}+\frac{1}{2}\left(\eta \cdot \xi^{\prime}-\xi \cdot \eta^{\prime}\right)\right)
$$

Accordingly (see $\left[\mathrm{Fo}\right.$ ), its unitary Schrödinger representation in $L^{2}\left(\mathbb{R}^{m}\right)$ is defined in the following way:

$$
(T(\eta, \xi) f)(x)=e^{2 \pi i \xi \cdot(\eta / 2+x)} f(x+\eta)
$$

This can be formally written as $T(\eta, \xi)=e^{2 \pi i(\eta \cdot P+\xi \cdot Q)}$, where $Q$ corresponds to the multiplication operator by $q$, and $P=(2 \pi i)^{-1} \nabla_{x}$. We have therefore taken $\hbar=(2 \pi)^{-1}$. It is evident that $T(\eta, \xi)$ preserves $L_{(k)}^{2}\left(T_{L}^{m}\right)$ : we denote $T^{(k)}(\eta, \xi)$ its restriction to that space.

We are now in position to define the quantization application. If $b$ is a pseudo-differential symbol of a given finite order (again [Fo] or [Sh]) on $\Lambda_{L}^{m}$, then

$$
\mathrm{Op}(b):=\frac{1}{L^{m}} \sum_{\xi \in\left(T_{L}^{m}\right)^{*}} \int_{\mathbb{R}^{m}} \widehat{b}(\eta, \xi) T(\eta, \xi) d \eta ;
$$

with $\widehat{b}$ possibly interpreted in distributional sense.

The restriction of this operator to the invariant space $L_{(k)}^{2}\left(T_{L}^{m}\right)$ will be once more denoted by $\mathrm{Op}(b)^{(k)}$. The above definition is nothing else than 
the standard Weyl quantization induced by the cylindrical Heisenberg group and subject to our choice of inverse Fourier transform (2.11). As a matter of fact, elementary algebraic manipulations yield the following explicit formula: for $f^{(k)} \in L_{(k)}^{2}\left(T_{L}^{m}\right)$,

$$
\left(\mathrm{Op}(b)^{(k)} f^{(k)}\right)(x)=\int_{\mathbb{R}^{m}} \int_{\mathbb{R}^{m}} b\left(\frac{x+y}{2}, p\right) e^{2 \pi i p \cdot(x-y)} f^{(k)}(y) d y d p .
$$

Remark that, since $f^{(k)} \notin \mathcal{S}\left(\mathbb{R}^{2 m}\right)$, a priori so this makes no sense even as an oscillatory integral. The sense we can give it is, again, distributional, as we know ([Sh]) that for pseudo-differential symbols, Op $(b): \mathcal{S}^{\prime} \rightarrow \mathcal{S}^{\prime}$. Notice also that, as usual, (2.15) implies that if $b$ depends only on one canonical variable, for instance $p$, then $\operatorname{Op}(b)=b(P)$ in the spectral-theoretic sense. In particular, if the quantum Hamiltonian is $H_{m}:=-\left(\frac{1}{8 \pi^{2}}\right) \Delta_{x}=\operatorname{Op}\left(\mathcal{H}_{m}\right)$, we have $\operatorname{Op}\left(e^{-\beta \mathcal{H}_{m}}\right)=e^{-\beta H_{m}}$.

We can now calculate the Weyl composition $a \sharp b$ of two symbols $a$ and $b$, i.e. the (unique) symbol such that $\mathrm{Op}(a \sharp b)=\mathrm{Op}(a) \mathrm{Op}(b)$. This is done by means of (2.14), remembering that $T(\eta, \xi)$ obeys the multiplication law of the Heisenberg group and that a symbol is obtained by its correspondent operator by substituting $e^{2 \pi i(p \cdot \eta+q \cdot \xi)}$ to $T(\eta, \xi)$ when the operator is in the form (2.14) - compare (2.11) to (2.14). The result, after some manipulations of the integrals, is - not surprisingly - an adaptment of the corresponding formula for the Euclidean space case $([\mathrm{Fo}], \S 2.1)$ :

$$
\begin{aligned}
& (a \sharp b)(p, q)= \\
= & \frac{1}{L^{2 m}} \sum_{\xi_{1}, \xi_{2}} \int \widehat{a}\left(\eta_{1}, \xi_{1}\right) \widehat{b}\left(\eta_{2}, \xi_{2}\right) e^{\pi i\left(\eta_{1} \cdot \xi_{2}-\xi_{1} \cdot \eta_{2}\right)} e^{2 \pi i\left[\left(\eta_{1}+\eta_{2}\right) \cdot p+\left(\xi_{1}+\xi_{2}\right) \cdot q\right]} d \eta_{1} d \eta_{2}= \\
= & \frac{1}{L^{2 m}} \sum_{\xi_{1}, \xi_{2}} \int a\left(p+\frac{\xi_{1}}{2}, q+q_{1}\right) b\left(p+\frac{\xi_{2}}{2}, q+q_{2}\right) e^{2 \pi i\left(\xi_{2} \cdot q_{1}-\xi_{1} \cdot q_{2}\right)} d q_{1} d q_{2} .
\end{aligned}
$$

Notice that the sum is carried over $\xi_{1}, \xi_{2} \in\left(T_{L}^{m}\right)^{*}$ and the integration over $q_{1}, q_{2} \in T_{L}^{m}$.

By the above formula we deduce that the Weyl composition has a property that may be called the quasi-tracial property:

$$
\int_{\Lambda_{L}^{m}}(a \sharp b)(p, q) d p d q=\int_{\Lambda_{L}^{m}} a(p, q) b(p, q) d p d q,
$$


even though we cannot hope to have the tracial property for some $L_{(k)}^{2}\left(T_{L}^{m}\right)$, i.e. (2.17) with $\operatorname{Tr}_{L_{(k)}^{2}\left(T_{L}^{m}\right)} \mathrm{Op}(a) \mathrm{Op}(b)$ on the 1.h.s., as explained in $\mathrm{dB}-\mathrm{G}$. Actually, as it will be clear in $\$ 3.1$, what appears in the l.h.s. is something resembling $\operatorname{Tr}_{L^{2}\left(\mathbb{R}^{m}\right)}$.

Given $f^{(k)}, g^{(k)} \in L_{(k)}^{2}\left(T_{L}^{m}\right)$, we define the Fourier-Wigner function relative to those two vectors as $V_{f^{(k)}, g^{(k)}}(\eta, \xi):=\left\langle f^{(k)}, T(\eta, \xi) g^{(k)}\right\rangle$. This is completely analogous to what is found in [F]]. The Wigner function $W_{f^{(k)}, g^{(k)}}$ is defined to be the (possibly distributional) Fourier transform of $V_{f^{(k)}, g^{(k)}}$ and thus, from (2.14),

$$
\left\langle f^{(k)}, \mathrm{Op}(b) g^{(k)}\right\rangle_{L_{(k)}^{2}\left(T_{L}^{m}\right)}=\int_{\Lambda_{L}^{m}} b(p, q) W_{f^{(k)}, g^{(k)}}(p, q) d p d q
$$

to be understood as $W_{f^{(k)}, g^{(k)}}$ being the distribution kernel of $b \mapsto\left\langle f^{(k)}, \mathrm{Op}(b) g^{(k)}\right\rangle$. Again the standard form for this "function" ([Fa]), calculated from (2.15) or from its very definition,

$$
W_{f^{(k)}, g^{(k)}}(p, q):=\int_{\mathbb{R}^{m}} e^{-2 \pi i p \cdot z} \overline{f^{(k)}(q-z / 2)} g^{(k)}(q+z / 2) d z .
$$

has to interpreted in the weak sense.

\section{Statement of the Results}

Suppose we have, $\forall L>0 ; m \in \mathbb{Z}, m \geq 1$ a measure space $\left(X_{L}^{m}, d \theta\right)$ ' ? and a family of states

$$
\left\{f_{\lambda}\right\}_{\lambda \in X_{L}^{m}} \subset \bigcup_{k \in[0,1 / L)^{m}} L_{(k)}^{2}\left(T_{L}^{m}\right)
$$

labeled by the index $\lambda$ ranging in $X_{L}^{m}$. Call $w_{\lambda}(p, q):=W_{f_{\lambda}, f_{\lambda}}(p, q)$, the Wigner function corresponding to $f_{\lambda}$.

Hypothesis. We suppose that

$$
\int_{X_{L}^{m}} w_{\lambda}(p, q) d \theta(\lambda) \equiv 1
$$

as a distribution on $\Lambda_{L}^{m}$.

\footnotetext{
${ }^{3}$ We are intentionally a little informal here, in order to keep the notation not to cumbersome. A better label for $d \theta$ would be $d \theta_{L}^{m}$. The same for $d \nu$ in the following.
} 
REMARK. Such family of states represent in this context the quantum substitute for the classical phase space. As a matter of fact, (3.2) says that the $\left\{f_{\lambda}\right\}$ are evenly distributed, as a whole, over $\Lambda_{L}^{m}$. Hence they play the role of "points". This is clearly seen in the case of the coherent states, perhaps the most remarkable example of states fulfilling the above hypothesis. They are introduced in $\S$ A.1 of the appendix. However, (3.2) can be restated by saying that we are given a set of states complete over all $L_{(k)}^{2}\left(T_{L}^{m}\right), k \in[0,1 / L)^{m}-$ see $\oint 3.1$.

Since $\left\|e^{-\beta H_{m}} f_{\lambda}\right\|^{2}=\left\langle f_{\lambda}, e^{-2 \beta H_{m}} f_{\lambda}\right\rangle$, 团 then (3.2) immediately implies

$$
\int_{X_{L}^{m}}\left\|e^{-\beta H_{m}} f_{\lambda}\right\|^{2} d \theta(\lambda)=\int_{\Lambda_{L}^{m}} e^{-2 \beta \mathcal{H}_{m}(p)} d p d q=L^{m}\left(\frac{\pi}{\beta}\right)^{m / 2} .
$$

We define:

$$
d \nu(\lambda):=\frac{\left\|e^{-\beta H_{m}} f_{\lambda}\right\|^{2} d \theta(\lambda)}{\int_{X_{L}^{m}}\left\|e^{-\beta H_{m}} f_{\lambda^{\prime}}\right\|^{2} d \theta\left(\lambda^{\prime}\right)}
$$

and

$$
g_{\lambda}:=\frac{e^{-\beta H_{m}} f_{\lambda}}{\left\|e^{-\beta H_{m}} f_{\lambda}\right\|}
$$

be the image under the quantum Gibbs measure of each of our states.

Definition 3.1 $a \in \mathcal{A}$ is said an asymptotic symbol if $\exists m_{0} \in \mathbb{N}, L_{0}>0$ such that $\forall m \geq m_{0}, L \geq L_{0}, a \circ \Pi_{L}^{m}$ is a pseudo-differential symbol over $\Lambda_{L}^{m}$.

REMARK. Notice that with such a definition, asymptotic symbols are rather rigid objects. In fact, fixed an $m \geq m_{0}$, take $L_{0} \leq L \leq L_{1}$. Now $\operatorname{Im}\left(\Pi_{L}^{m}\right) \subseteq \operatorname{Im}\left(\Pi_{L_{1}}^{m}\right) \subseteq \Lambda_{\infty}$. So $a \circ \Pi_{L}^{m}$ is just the restriction of $a \circ \Pi_{L_{1}}^{m}$ to $\Lambda_{L}^{m}$. But also $a \circ \Pi_{L}^{m}$, in order to be a symbol must be $C^{\infty}$ and $T_{L}^{m}$-periodic. This means that $\forall i=1, \ldots, m$,

$$
\begin{aligned}
\left(a \circ \Pi_{L_{1}}^{m}\right)(\ldots,-L / 2, \ldots) & =\left(a \circ \Pi_{L_{1}}^{m}\right)(\ldots,+L / 2, \ldots) ; \\
\frac{d}{d q_{i}}\left(a \circ \Pi_{L_{1}}^{m}\right)(\ldots,-L / 2, \ldots) & =\frac{d}{d q_{i}}\left(a \circ \Pi_{L_{1}}^{m}\right)(\ldots,+L / 2, \ldots),
\end{aligned}
$$

\footnotetext{
${ }^{4}$ Again a remark about the notation. A more formal symbol for the scalar product would be $\langle\cdot, \cdot\rangle_{L_{(k)}^{2}\left(T_{L}^{m}\right)}$ where $k=k(\lambda)$ is the uniquely determined $k \in[0,1 / L)^{m}$ such that the arguments are in $L_{(k)}^{2}\left(T_{L}^{m}\right)$. Since the scalar products have the same structure on each $L_{(k)}^{2}\left(T_{L}^{m}\right)$ we will drop that subscript.
} 
where $(\ldots, \pm L / 2, \ldots)$ stands for $\left(p_{1}, \ldots, p_{m}, q_{1}, \ldots, q_{i-1}, \pm L / 2, q_{i+1}, \ldots, q_{m}\right)$. Thus, parity arguments imply that for every $\left|q_{i}\right| \geq L_{0} / 2$

$$
\frac{d}{d q_{i}}\left(a \circ \Pi_{L_{1}}^{m}\right)\left(\ldots, q_{i}, \ldots\right)=0
$$

Hence, for a fixed large $m,\left(a \circ \prod_{L_{1}}^{m}\right)$ is a constantwise continuation of $\left(a \circ \prod_{L_{0}}^{m}\right)$, and the former is completely determined by the latter. This also explain we had to ask for asymptotic symbols: one could not request the above property to hold $\forall m \geq 0, L \geq L_{0}$. Examples of such functions are to be found in the families $\mathcal{B}^{(n)}$ defined after Lemma 4.2 in $\S$.

We are now ready to state the theorems. For any operator $A$ acting over $L^{2}\left(\mathbb{R}^{m}\right)$, define:

$$
\begin{aligned}
\Theta^{m}[t](A) & :=e^{2 \pi i t H_{m}} A e^{-2 \pi i t H_{m}} \\
\Xi^{m}[T](A) & :=\frac{1}{2 T} \int_{-T}^{T} \Theta^{m}[t](A) d t .
\end{aligned}
$$

The quantum mixing property reads:

Theorem 3.2 Suppose $a, b$ asymptotic symbols in $L^{2}\left(\Lambda_{\infty}, P_{\rho, 2 \beta}\right)$ and denote

$$
I(t, L, m):=\int_{X_{L}^{m}}\left\langle g_{\lambda}, \Theta^{m}[t]\left(\mathrm{Op}\left(a \circ \Pi_{L}^{m}\right)\right) \mathrm{Op}\left(b \circ \Pi_{L}^{m}\right) g_{\lambda}\right\rangle d \nu(\lambda) .
$$

Then:

$$
\lim _{|t| \rightarrow \infty} \lim _{\substack{m, L \rightarrow \infty \\ m / L \rightarrow \rho}} I(t, L, m)=P_{\rho, 2 \beta}(a) P_{\rho, 2 \beta}(b) .
$$

As regards the quantum ergodicity,

Theorem 3.3 Let a be an asymptotic symbol in $L^{2}\left(\Lambda_{\infty}, P_{\rho, 2 \beta}\right)$. Let

$$
J(T, L, m):=\int_{X_{L}^{m}}\left\|\left(\Xi^{m}[T]\left(\mathrm{Op}\left(a \circ \Pi_{L}^{m}\right)\right)-P_{\rho, 2 \beta}(a)\right) g_{\lambda}\right\|^{2} d \nu(\lambda) .
$$

Then, for all $L, m$, the operator

$$
\Xi^{m}[\infty]\left(\mathrm{Op}\left(a \circ \Pi_{L}^{m}\right)\right):=\lim _{T \rightarrow \infty} \Xi^{m}[T]\left(\mathrm{Op}\left(a \circ \Pi_{L}^{m}\right)\right)
$$


exists in the domain of $\operatorname{Op}\left(a \circ \Pi_{L}^{m}\right)$ and

$$
\lim _{\substack{m, L \rightarrow \infty \\ m / L \rightarrow \rho}} J(\infty, L, m)=0 .
$$

Furthermore, if a is also bounded, then the two limits can be inverted:

$$
\lim _{T \rightarrow \infty} \lim _{\substack{m, L \rightarrow \infty \\ m / L \rightarrow \rho}} J(T, L, m)=0 .
$$

REMARK. The interchange of the time limit with the thermodynamical limit, in the above theorem, is remarkable. The fact that the time average can be taken before the thermodynamical limit can be described saying that the finite-particle system (the "real" one) is quasi-ergodic, for very large $L$ and $m$; that is, the time average of any decent function is close, in measure, to a constant. This is a feature of the classical ideal gas which has nothing to do with quantum mechanics. It is rather a consequence of the kinematic chaos and the restriction to symmetric observables, as anticipated in \$1, remark (i). This can be seen quite easily, due to the integrability of the motion: time averaging means almost everywhere averaging over a torus. The invariant tori here are the sets $\{p\} \times T_{L}^{m} \in \Lambda_{L}^{m}$, and so the time average of $a(p, q)$ is simply $\int a(p, q) d q$; the invariant functions would depend on $p$ only. Those functions, however, are requested to be symmetric and thus they cannot concentrate around a torus if they do not concentrate around all "symmetric tori" as well. An example is

$$
a\left(p_{1}, \ldots, p_{m}\right)= \begin{cases}1 & \text { if } p_{i} \in \Gamma \forall i=1, \ldots, m \\ 0 & \text { otherwise }\end{cases}
$$

where $\Gamma$ is a Borel set of $\mathbb{R}$. Now the kinematic chaos effect comes: at the thermodynamical limit, the support of this function, which is the probability to find all the particles having momenta in $\Gamma$, is exponentially small.

We can compare this to the situation one has for the harmonic chain, as shown in the companion paper G-M]. In that case, there is no requirement on the observables. The fact that they cannot concentrate over invariant tori is instead due to the assumptions on the coupling matrix, which shuffles the tori at the infinite-particle limit.

An even clearer reason for referring to Theorem 3.3 as quantum ergodicity comes from the following: 
Corollary 3.4 Assume a bounded asymptotic symbol. Then, $\forall \epsilon>0$, set

$K(\epsilon, T, L, m):=\nu\left(\left\{\lambda \in X_{L}^{m}||\left\langle g_{\lambda}, \Xi^{m}[T]\left(\mathrm{Op}\left(a \circ \Pi_{L}^{m}\right)\right) g_{\lambda}\right\rangle-P_{\rho, 2 \beta}(a) \mid>\epsilon\right\}\right)$.

Then

$$
\lim _{T \rightarrow \infty} \lim _{\substack{m, L \rightarrow \infty \\ m / L \rightarrow \rho}} K(\epsilon, T, L, m)=\lim _{\substack{m, L \rightarrow \infty \\ m / L \rightarrow \rho}} \lim _{T \rightarrow \infty} K(\epsilon, T, L, m)=0 .
$$

This can be phrased as follows. Call $(T, \epsilon)$-exceptional initial states those states $g_{\lambda}$ for which the quantum expectation of the $T$-time average is greater than $\epsilon$. Then the claim is that the measure of the $(T, \epsilon)$-exceptional initial states vanishes when the thermodynamical limit and the time limit are achieved.

Proof of Corollary 3.4. Easy consequence of Theorem 3.3, using a Cauchy-Schwartz inequality.

Q.E.D.

\subsection{The Quantum Gibbs State}

The results just formulated can be given a compact form, within the realm of the $C^{*}$ dynamical systems theory. It is beyond the purpose of this paper to go deep into that, so we do not outline the main notions of such a theory, hoping that the statements in this section are self-explanatory. However, a brief survey is given in G-M], Appendix 2. Here we just observe that the relations we will write are included in such a general frame. The interested reader is referred to $[\bar{B}-\mathrm{R}]$ for complete details, and to $[\mathrm{B}]$ for a recent wellorganized review.

Consider $\mathcal{L}_{L}^{m}$, the space of all operators on $L^{2}\left(\mathbb{R}^{m}\right)$ which are invariant and bounded over all the fibers $L_{(k)}^{2}\left(T_{L}^{m}\right)$. This is a $C^{*}$-algebra when endowed with the usual operator norm. Associated to this algebra we define the Heisenberg dynamics given by $\Theta^{m}[t](A)$ as in (3.9), and the quantum Gibbs state expressed by

$$
\omega_{\beta, L}^{m}(A):=\frac{\int_{[0,1 / L)^{m}} \operatorname{Tr}_{L, m, k}\left(A e^{-\beta H_{m}}\right) d k}{\int_{[0,1 / L)^{m}} \operatorname{Tr}_{L, m, k}\left(e^{-\beta H_{m}}\right) d k},
$$

where $\operatorname{Tr}_{L, m, k}$ denotes the trace over $L_{(k)}^{2}\left(T_{L}^{m}\right)$. This functional is clearly normalized 9 and invariant for the ${ }^{*}$-automorphism $\Theta^{m}[t]$. Actually, it turns

\footnotetext{
${ }^{5}$ Here normalized means $\omega_{\beta, L}^{m}(\mathbf{1})=1$.
} 
out to be a KMS state with parameter $\beta$ over the $W^{*}$ dynamical system $\left(\mathcal{L}_{L}^{m}, \Theta^{m}[t], \omega_{\beta, L}^{m}\right)$.

Now consider, for each value of $k \in[0,1 / L)^{m}$, the standard Fourier basis $\left\{e_{\alpha}^{(k)}\right\}$ as defined in $\$ 2.1$. Next consider the family of all such vectors, labeled by the index $\lambda:=(\alpha, k) \in X_{L}^{m}=:\left(T_{L}^{m}\right)^{*} \times[0,1 / L)^{m}$. Endow $X_{L}^{m}$ with the measure

$$
d \theta(\alpha, k):=L^{m} \sum_{\xi \in\left(T_{L}^{m}\right)^{*}} \delta(\alpha-\xi) d \alpha d k .
$$

Such a family satisfies hypothesis (3.2). In fact, calling $w_{\alpha, k}$ the Wigner function relative to $e_{\alpha}^{(k)}$, a straightforward computation from (2.19) yields

$$
w_{\alpha, k}(p, q)=\frac{1}{L^{m}} \delta(p-(\alpha+k)) .
$$

Integrating this in $d \theta(\alpha, k)$ we obtain (3.2). Thus, define, as it is done in at the beginning of this section, $g_{\alpha, k}:=e^{-\beta H_{m}} e_{\alpha}^{(k)} /\left\|e^{-\beta H_{m}} e_{\alpha}^{(k)}\right\|$. By definition (3.12), if $A \in \mathcal{L}_{L}^{m}$, we have 9

$$
\begin{aligned}
\omega_{2 \beta, L}^{m}(A) & =\frac{\int d k \sum_{\alpha}\left\langle e^{-\beta H_{m}} e_{\alpha}^{(k)}, A e^{-\beta H_{m}} e_{\alpha}^{(k)}\right\rangle}{\int d k \sum_{\alpha}\left\|e^{-\beta H_{m}} e_{\alpha}^{(k)}\right\|^{2}}= \\
& =\int_{X_{L}^{m}}\left\langle g_{\alpha, k}, A g_{\alpha, k}\right\rangle d \nu(\alpha, k),
\end{aligned}
$$

with $d \nu$ defined as in (3.4). On the other hand, if one calls in a natural way $A_{L}^{m}:=\operatorname{Op}\left(a \circ \Pi_{L}^{m}\right)$, then a simple argument which is better explained in the following (see formulæ (4.2) and (4.3)) gives

$$
\omega_{2 \beta, L}^{m}\left(A_{L}^{m}\right)=\int_{\Lambda_{L}^{m}}\left(a \circ \Pi_{L}^{m}\right)(p, q)\left[e^{-2 \beta \mathcal{H}_{m}(p)} d p d q\right]_{N} .
$$

Proposition 2.1 immediately yields

$$
\lim _{\substack{m, L \rightarrow \infty \\ m / L \rightarrow \rho}} \omega_{2 \beta, L}^{m}\left(A_{L}^{m}\right)=P_{\rho, 2 \beta}(a) .
$$

Hence, if $a, b$ are asymptotic symbols, we have just proved that Theorem 3.2 can be rewritten as

$$
\lim _{|t| \rightarrow \infty} \lim _{\substack{m, L \rightarrow \infty \\ m / L \rightarrow \rho}} \omega_{2 \beta, L}^{m}\left(\Theta^{m}[t]\left(A_{L}^{m}\right) B_{L}^{m}\right)=\lim _{\substack{m, L \rightarrow \infty \\ m / L \rightarrow \rho}} \omega_{2 \beta, L}^{m}\left(A_{L}^{m}\right) \cdot \lim _{\substack{m, L \rightarrow \infty \\ m / L \rightarrow \rho}} \omega_{2 \beta, L}^{m}\left(B_{L}^{m}\right) .
$$

\footnotetext{
${ }^{6}$ Notice that we are using $2 \beta$ instead of $\beta$ in the remainder.
} 
In the same spirit, Theorem 3.3 becomes

$$
\begin{aligned}
& \lim _{T \rightarrow \infty} \lim _{\substack{m, L \rightarrow \infty \\
m / L \rightarrow \rho}} \omega_{2 \beta, L}^{m}\left(\left(\Xi^{m}[T]\left(A_{L}^{m}\right)\right)^{2}\right)= \\
= & \lim _{\substack{m, L \rightarrow \infty \\
m / L \rightarrow \rho}} \omega_{2 \beta, L}^{m}\left(\left(\Xi^{m}[\infty]\left(A_{L}^{m}\right)\right)^{2}\right)= \\
= & \lim _{\substack{m, L \rightarrow \infty \\
m / L \rightarrow \rho}}\left(\omega_{2 \beta, L}^{m}\left(A_{L}^{m}\right)\right)^{2},
\end{aligned}
$$

REMARK. We have not defined an algebra of quantum observables for the infinite-particle system, limiting ourselves to deal with finite dimensions and to take a thermodynamical limit afterwards (see also comment 3 below). Had we introduced such a mathematical framework, then relations (3.18) and (3.19), for the state $\lim _{\substack{m, L \rightarrow \infty \\ m / L \rightarrow \infty}} \omega_{2 \beta, L}^{m}$, would be contained in the general set of chaoticity notions in $C^{*}$ dynamical system theory (see [B], Definitions $4.42,4.43)$. I

Some comments concerning the above reformulation of the theorems as compared to [G-M]:

1. (3.18) is completely analogous to statement (1.10) in [G-M]. That is, the quantum mixing, forbidden in the finite-particle frame by the quasiperiodicity of the Heisenberg evolution, regardless the dynamics of the classical flow (see $\S[$ ), is restored at the thermodynamical limit.

2. A formulation of the ergodicity similar to (1.9) in [G-M] has not been chosen in this context because of the technicalities it would require. The coherent states we have here (see appendix, $\S$ A.1) are indexed by $\lambda \in T_{L}^{m} \times \mathbb{R}^{m} \times[0,1 / L)^{m}=: X_{L}^{m}$, which is not exactly the classical phase space $\Lambda_{L}^{m}$. However, one could explicitly calculate $d \nu$ over $X_{L}^{m}$, for particular choices of the coherent states, and find a limit measure space - say - $(X, d \nu)$, but this turns out to be rather cumbersome and possibly misleading. Understandably, though, (3.19) and especially Corollary 3.4 carry the same physical meaning as the mentioned result.

3. As already exploited, we are able here to state the ergodicity results with a commutation of the limits.

\footnotetext{
${ }^{7}$ We are not granted, in principle, all the equivalent ergodicity and mixing properties recalled in that reference, since we have not proved asymptotic commutativity.
} 
Ergodic Properties of the Quantum Ideal Gas ...

4. As emphasized in [G-M], $\S 1$, Remark 2, the techniques we use to prove the quantum ergodic properties at the thermodynamical limit, have the useful outcome to show that the r.h.sides of (3.19) and (3.18) are the expected classical Gibbs averages. This is why we have formulated Theorems 3.3 and 3.2 in the first place.

5. More importantly, here, results (3.18) and (3.19) were not known, at least to us.

\section{The Proofs.}

The first key fact is the following

Lemma 4.1 For every symbol c defined on $\Lambda_{L}^{m}$,

$$
e^{2 \pi i t H_{m}} \mathrm{Op}(c) e^{-2 \pi i t H_{m}}=\mathrm{Op}\left(c \circ \phi_{L}^{m}[t]\right)
$$

This is true since we are dealing with a linear flow. This property of linear flows - sometimes referred to as the exact Egorov Theorem for the evolution canonical transformation - dates back at least to Van Hove and is valid only for Weyl quantization, whose restriction to $L$-periodic symbols we are now using. Anyway, for the sake of convenience, a direct proof is found in $\$$ A.2 of the appendix.

In our case, applying this lemma to $a \circ \prod_{L}^{m}$ and using the remark in formula (2.4), we have

$$
e^{2 \pi i t H_{m}} \mathrm{Op}\left(a \circ \Pi_{L}^{m}\right) e^{-2 \pi i t H_{m}}=\mathrm{Op}\left(a \circ \phi_{\infty}[t] \circ \Pi_{L}^{m}\right) .
$$

\subsection{Proof of Theorem 3.2.}

In view of the above relation we call $a_{t}:=a \circ \phi_{\infty}[t]$. In the rest of this proof, whenever there is no confusion, we denote by a quote the immersion application from $\Lambda_{L}^{m}$ to $\Lambda_{\infty}$. Hence $a^{\prime}:=a \circ \Pi_{L}^{m}$ and so on. In other words, $a^{\prime}$ is just our observable $a$ looked at in the finite dimensional phase space $\Lambda_{L}^{m}$. By (3.2) and (3.4), the definition of $I$, in the statement of the theorem, yields

$$
I(t, L, m)=\frac{\int_{\Lambda_{L}^{m}}\left(e^{-\beta \mathcal{H}_{m}} \sharp a_{t}^{\prime} \sharp b^{\prime} \sharp e^{-\beta \mathcal{H}_{m}}\right) d p d q}{\int_{\Lambda_{L}^{m}} e^{-2 \beta \mathcal{H}_{m}} d p d q} .
$$


Using twice (2.17) - once to permutate cyclically the factors in (4.2) and once to remove one of the $\sharp$ signs - leads to

$$
I(t, L, m)=\int_{\Lambda_{L}^{m}}\left(a_{t}^{\prime} \sharp b^{\prime}\right)(p, q)\left[e^{-2 \beta \mathcal{H}_{m}(p)} d p d q\right]_{N},
$$

since obviously $e^{-\beta \mathcal{H}_{m}} \sharp e^{-\beta \mathcal{H}_{m}}=e^{-2 \beta \mathcal{H}_{m}}$. We further denote by $\mu_{L}^{m}$ the classical Gibbs measure (at inverse temperature $2 \beta$ ) over $\Lambda_{L}^{m}: \mu_{L}^{m}(p, q):=$ $L^{-m}(\pi / \beta)^{-m / 2} e^{-\beta p^{2}}$, and by $\breve{\mu}^{m}$ its component in the $p$-space: $\breve{\mu}^{m}(p):=$ $(\pi / \beta)^{-m / 2} e^{-\beta p^{2}}$.

In view of (2.16), (4.3) becomes, after some elementary but tedious rearrangements of the nested integrals,

$$
I(t, L, m)=\int_{\Lambda_{L}^{m}}\left[a_{t}^{\prime}\left(b^{\prime} *_{L} \Phi_{L}^{m}\right)\right](p, q) d \mu_{L}^{m}(p, q)
$$

where

$$
\Phi_{L}^{m}(p, q)=e^{\beta p^{2}} \frac{1}{L^{m}} \sum_{\xi \in\left(T_{L}^{m}\right)^{*}} e^{-\beta(p-\xi / 2)^{2}} e^{2 \pi i \xi \cdot q},
$$

and $*_{L}$ means convolution in the $q$-variable on $T_{L}^{m}$. A particular care must be taken here about this convolution on a torus, in order to prevent mistakes: see $\S$ A.3.

Thus $\Phi_{L}^{m}$ is completely factorizable, with $\Phi_{L}$ being a natural symbol for each of his factor: if $f\left(p_{1}, \ldots, p_{m}, q_{1}, \ldots, q_{m}\right)=f_{1}\left(p_{1}, q_{1}\right) \cdots f_{m}\left(p_{m}, q_{m}\right)$, then

$$
\left(f *_{L} \Phi_{L}^{m}\right)(p, q)=\left(f_{1} *_{L} \Phi_{L}\right)\left(p_{1}, q_{1}\right) \cdots\left(f_{m} *_{L} \Phi_{L}\right)\left(p_{m}, q_{m}\right)
$$

This property will be useful in the following. Also, if 1 is the function on $\Lambda_{L}^{m}$ identically equal to 1 ,

$$
1 *_{L} \Phi_{L}^{m}=1
$$

We are going to prove the statement of the theorem, starting from (4.4), for $b$ in a dense subspace of $L^{2}\left(\Lambda_{\infty}, P_{\rho, 2 \beta}\right)$. To accomplish that we need to make the following construction.

Fix a positive integer $n$ and consider the function $\beta(p, q) \in C_{0}^{\infty}\left(\mathbb{R}^{2 n}\right) \S$, that is, infinitely differentiable functions with compact support. For $m>n$

\footnotetext{
${ }^{8}$ Not to be confused with the inverse temperature $\beta$, a fixed parameter throughout this paper.
} 
define the application

$$
N_{L, m}^{(n)}(\beta)\left(p_{1}, \ldots, p_{m}, q_{1}, \ldots, q_{m}\right):=\sum_{j_{1}=1}^{m} \cdots \sum_{j_{n}=1}^{m} \beta\left(p_{j_{1}}, \ldots, p_{j_{n}}, q_{j_{1}}, \ldots, q_{j_{n}}\right) .
$$

So $N_{L, m}^{(n)}(\beta)$ is a function defined on $\mathbb{R}^{2 m}$, and thus in particular on $\Lambda_{L}^{m}$. The use of this application is explained by next

Lemma 4.2 If $\beta(p, q) \in \mathcal{S}\left(\mathbb{R}^{2 n}\right)$ 马 then there exists a function $b \in L^{2}\left(\Lambda_{\infty}, P_{\rho, 2 \beta}\right)$ s.t. $N_{L, m}^{(n)}(\beta)=b \circ \Pi_{L}^{m}$. Plus, the following properties hold:

$$
\begin{gathered}
\int_{\Lambda_{L}^{m}}\left|\left(b \circ \Pi_{L}^{m}\right)(p, q)\right|^{2} d \mu_{L}^{m}(p, q) \leq m^{2 n} \int_{\mathbb{R}^{2 n}}\left|\beta\left(p^{\prime}, q^{\prime}\right)\right|^{2} d \mu_{L}^{n}\left(p^{\prime}, q^{\prime}\right) \\
\int_{\Lambda_{L}^{m}}\left(b \circ \Pi_{L}^{m}\right)(p, q) d \mu_{L}^{m}(p, q)=\frac{m !}{L^{m}(m-n) !} \int_{\mathbb{R}^{2 n}} \beta\left(p^{\prime}, q^{\prime}\right) d \breve{\mu}^{n}\left(p^{\prime}\right) d q^{\prime} .
\end{gathered}
$$

Proof of Lemma 4.2. The first inequality simply follows by definition (4.8): we have $m^{2 n}$ integrals over $\Lambda_{L}^{m}$. To the cross-term integrals we apply Cauchy-Schwartz in order to obtain $m^{2 n}$ terms equal to $\|\beta\|_{L^{2}\left(\Lambda_{L}^{m}\right)}^{2}$. These get reduced to integrals over $\Lambda_{L}^{n}$, since the measure is decomposable and the integrand functions depend only on $2 n$ variables; finally they are extended to all of $\mathbb{R}^{2 n}$.

To prove the rest we approximate $\beta$ with suitably chosen indicator functions over $\mathbb{R}^{2 n}$. More precisely take two sufficiently fine partitions of $\mathbb{R}$, $\left\{\Gamma_{j}\right\},\left\{\Delta_{\ell}\right\} \subset \mathcal{B}(\mathbb{R})$ with $\sup _{j, \ell}\left\{\breve{\mu}\left(\beta_{j}\right),\left|\Delta_{\ell}\right|\right\}$ small. Tot Let $\chi_{j_{1}, \ldots, j_{n}, \ell_{1}, \ldots, \ell_{n}}\left(p^{\prime}, q^{\prime}\right)$ be the indicator function of the set $\Gamma_{j_{1}} \times \cdots \times \Gamma_{j_{n}} \times \Delta_{\ell_{1}} \times \cdots \times \Delta_{\ell_{1}}$ and approximate $\beta$ with $\beta_{a}:=\sum c_{j_{1}, \ldots, \ell_{n}} \chi_{j_{1}, \ldots, \ell_{n}}$. So

$$
N_{L, m}^{(n)}\left(\beta_{a}\right)(p, q)=\sum_{j_{1}, \ldots, \ell_{n}} c_{j_{1}, \ldots, \ell_{n}} N_{L, m}^{(n)}\left(\chi_{j_{1}, \ldots, \ell_{n}}\right)(p, q) .
$$

Since $\chi_{j_{1}, \ldots, \ell_{n}}$ is completely factorizable, then it is easy to realize, by definition (4.8), that $N_{L, m}^{(n)}\left(\chi_{j_{1}, \ldots, \ell_{n}}\right)(p, q)=N_{L, m}^{(1)}\left(\chi_{\Gamma_{j_{1}} \times \Delta_{\ell_{1}}}\right) \cdots N_{L, m}^{(1)}\left(\chi_{\Gamma_{j_{n}} \times \Delta_{\ell_{n}}}\right)$. We see that $N_{L, m}^{(1)}\left(\chi_{\Gamma \times \Delta}\right)(p, q)$ takes integer values between 0 and $m$. Specifically if counts the number of particles in the configuration $(p, q) \in \Lambda_{L}^{m}$ whose

\footnotetext{
${ }^{9}$ This denotes the Schwartz class.

${ }^{10}|\cdot|$ denotes the Lebesgue measure.
} 
momentum is contained in $\Gamma$ and whose coordinate in $\Delta$. 凹 So $N_{L, m}^{(1)}\left(\chi_{\Gamma \times \Delta}\right)=$ $N_{\Gamma \times \Delta} \circ \Pi_{L}^{m}$, where $N_{\Gamma \times \Delta}: \Lambda_{\infty} \rightarrow \mathbb{N}$ is defined by

$$
N_{\Gamma \times \Delta}(p, q):=\#\{x \in q \cap \Delta \mid p(x) \in \Gamma\},
$$

where, with sloppy notation, $(p, q)$ denotes a point in $\Lambda_{\infty}$. Recalling what we said in $\$ 2.1$, this function obviously belongs to $\mathcal{A}$ : see in particular (2.7) and comments thereby. Therefore so does every finite product of similar functions. Looking at (4.11), and subsequent comments, this proves that there exists a $b_{a} \in \mathcal{A}$ such that $\beta_{a}=b_{a} \circ \Pi_{L}^{m}$. The analogous statement holds for $\beta$ as well, by density.

Let us go over to the proof of (4.10). Fix a sequence $(j, \ell):=\left(j_{1}, \ldots, \ell_{n}\right)$ like those we have in formula (4.11) and fix $n$ integers $k:=\left(k_{1}, \ldots, k_{n}\right)$ such that $k_{1}+\ldots+k_{n} \leq m$. Now consider the set $A_{j, \ell}^{(k)}:=\left\{N_{L, m}^{(1)}\left(\chi_{\Gamma_{j_{i} \times \Delta_{\ell_{i}}}}\right)=\right.$ $\left.k_{i} ; \forall i=1, \ldots, n\right\} \in \Lambda_{L}^{m}$, i.e. the set of the configurations having $k_{1}$ particles in $\Gamma_{j_{1}} \times \Delta_{\ell_{1}}, k_{2}$ particles in $\Gamma_{j_{2}} \times \Delta_{\ell_{2}}$, and so on. Notice that (use some combinatorics and, anyway, refer to $[\mathrm{S}]$ )

$\mu_{L}^{m}\left(A_{j, \ell}^{(k)}\right)=\frac{m !}{\left(m-\sum k_{i}\right) !}\left[\prod_{i=1}^{n} \frac{1}{k_{i} !}\left(\frac{\left|\Delta_{\ell_{i}}\right|}{L} \breve{\mu}\left(\Gamma_{j_{i}}\right)\right)^{k_{i}}\right]\left(1-\sum_{i=1}^{n} \frac{\left|\Delta_{\ell_{i}}\right|}{L} \breve{\mu}\left(\Gamma_{j_{i}}\right)\right)^{m-\sum k_{i}}$

We have seen that $N_{L, m}^{(n)}\left(\chi_{j_{1}, \ldots, \ell_{n}}\right)=\sum_{k} k_{1} \cdots k_{n} A_{j, \ell}^{(k)}$. So, when the initially chosen partition is fine, $\mu_{L}^{m}\left(\chi_{j_{1}, \ldots, \ell_{n}}\right)=m ! /\left(L^{m}(m-n) !\right) \prod_{i}\left|\Delta_{\ell_{i}}\right| \breve{\mu}\left(\Gamma_{j_{i}}\right)+$ $o\left(\left|\Delta_{\ell_{i}}\right|, \breve{\mu}\left(\Gamma_{j_{i}}\right)\right)$. Looking back at (4.11) this proves that 4.10) holds with negligible errors for $\beta_{a}$ and thus is exact for $\beta$.

Q.E.D.

Let us call $\mathcal{B}^{(n)} \in \mathcal{A}$ the space of functions $b$ granted by Lemma 4.2 when $\beta \in C_{0}^{\infty}\left(\mathbb{R}^{2 n}\right)$. From now on we will suppose $b \in \mathcal{B}^{(n)}$, so that $b^{\prime}:=b \circ \Pi_{L}^{m}=$ $N_{L, m}^{(n)}(\beta)$. In so doing we will be proving Theorem 3.2 for $b \in \oplus_{\text {finite }} \mathcal{B}^{(n)}$. But this is dense in $L^{2}\left(\Lambda_{\infty}, P_{\rho, 2 \beta}\right)$ since, looking at the proof of Lemma 4.2, the closure of $\mathcal{B}^{(n)}$ contains the product of $n$ functions like $N_{\Gamma \times \Delta}$. This means that in the algebra $\overline{\left(\oplus \mathcal{B}^{(n)}\right)}$ we are able to find the indicator functions of the sets $N_{\Gamma \times \Delta}^{-1}(n), \forall n \in \mathbb{N}$. But these generate $\mathcal{A}$ (look at 2.7 and refer to G-L-A and B]).

Under the above assumption, we go back to (4.4): since $b^{\prime}=N_{L, m}^{(n)}(\beta)$,

${ }^{11}$ This explains why we have chosen such a notation for $N_{L, m}^{(n)}$. 
then $b^{\prime} *_{L} \Phi_{L}^{m}=N_{L, m}^{(n)}\left(\beta *_{L} \Phi_{L}^{n}\right) \llbracket$ because of the mentioned properties of $\Phi_{L}^{m}$ (see (4.5) to (4.7)). If we denote by $\gamma_{L}:=\beta *_{L} \Phi_{L}^{n}$, it is obvious that $\gamma_{L} \in \mathcal{S}\left(\mathbb{R}^{2 n}\right)$ and so $N_{L, m}^{(n)}\left(\gamma_{L}\right)=c_{L}^{\prime}$ for some $c_{L} \in L^{2}\left(\Lambda_{\infty}, P_{\rho, 2 \beta}\right)$, by Lemma 4.2. This allows us to rewrite (4.4) as

$$
I(t, L, m)=\left\langle a_{t}^{\prime}, c_{L}^{\prime}\right\rangle_{L^{2}\left(\Lambda_{L}^{m}, \mu_{L}^{m}\right)} .
$$

If we are able to find a limit for $c_{L}$ then we are done with the cumbersome part of this proof. To this goal, we formulate the following

Lemma 4.3 There exists a $\gamma_{\infty} \in \mathcal{S}\left(\mathbb{R}^{2 n}\right)$ such that

$$
\left\|\gamma_{\infty}-\gamma_{L}\right\|_{L^{2}\left(\Lambda_{L}^{n}, \mu_{L}^{n}\right)}^{2}=\mathcal{O}\left(L^{-\infty}\right) .
$$

Furthermore

$$
\int_{\mathbb{R}^{2 n}} \gamma_{\infty}\left(p^{\prime}, q^{\prime}\right) d \breve{\mu}^{n}\left(p^{\prime}\right) d q^{\prime}=\int_{\mathbb{R}^{2 n}} \beta\left(p^{\prime}, q^{\prime}\right) d \breve{\mu}^{n}\left(p^{\prime}\right) d q^{\prime} .
$$

The proof of this lemma is found in $\S$ A.4.

In analogy with the above notations we call $c_{\infty}$ the observable in $L^{2}\left(\Lambda_{\infty}, P_{\rho, 2 \beta}\right)$ obtained applying Lemma 4.2 to $\gamma_{\infty}$. Comparing now (4.15) in Lemma 4.3 with (4.9) we deduce that

$$
\lim _{\substack{m, L \rightarrow \infty \\ m / L \rightarrow \rho}}\left\|c_{\infty}^{\prime}-c_{L}^{\prime}\right\|_{L^{2}\left(\Lambda_{L}^{m}, \mu_{L}^{m}\right)}^{2}=0 .
$$

Dropping for the sake of simplicity the subscript in the scalar product notation, this means that, when $m, L \rightarrow \infty, m / L \rightarrow \rho$,

$$
\begin{aligned}
& \left|\left\langle a_{t}^{\prime}, c_{L}^{\prime}\right\rangle-P_{\rho, 2 \beta}\left(a_{t} c_{\infty}\right)\right| \leq \\
\leq & \left\|a_{t}^{\prime}\right\|^{2}\left\|c_{L}^{\prime}-c_{\infty}^{\prime}\right\|^{2}+\left|\mu_{L}^{m}\left(a_{t}^{\prime} c_{\infty}^{\prime}\right)-P_{\rho, 2 \beta}\left(a_{t} c_{\infty}\right)\right| \rightarrow 0,
\end{aligned}
$$

because of Proposition 2.1. Now we use the other main ingredient of this proof, i.e. the classical result, Theorem 2.2. We obtain

$$
\lim _{|t| \rightarrow \infty} \lim _{\substack{m, L \rightarrow \infty \\ m / L \rightarrow \rho}} I(t, L, m)=P_{\rho, 2 \beta}(a) P_{\rho, 2 \beta}\left(c_{\infty}\right) .
$$

\footnotetext{
${ }^{12}$ Where this time $*_{L}$ means convolution in the $q$-variable over $T_{L}^{n}$. We warn the reader again about the possible confusion arising from the fact that $\beta(p, q)$ is defined on $\mathbb{R}^{2 n}$. When in a $*_{L}$-convolution, it has to be considered as restricted to $[-L / 2, L / 2)^{n}$ and periodic according to the identification $[-L / 2, L / 2)^{n} \simeq T_{L}^{n}$. See again $\S$ A.3.
} 
Now, using the integrals of $\gamma_{\infty}$ and $\beta$ to compare the integrals of $c_{\infty}$ and $b$ (apply (4.16) into (4.10)), we see that $\mu_{L}^{m}\left(c_{\infty}^{\prime}\right)=\mu_{L}^{m}\left(b^{\prime}\right)$. Taking the limits, $P_{\rho, 2 \beta}\left(c_{\infty}\right)=P_{\rho, 2 \beta}(b)$, which, together with the last relation, completes the proof.

Q.E.D.

\subsection{Proof of Theorem 3.3.}

First of all it has to be noticed that both statements of Theorem 3.3 (respectively relation (3.19) ) cannot be derived so trivially from Theorem 3.2 (resp. (3.18)). This will be seen below in each case.

We borrow the notation from the previous proof: so, for example, $a_{t}^{\prime}:=$ $a \circ \phi_{\infty}[t] \circ \Pi_{L}^{m}$. Also, let $a_{T}^{\prime}:=(1 / 2 T) \int_{-T}^{T} a_{t}^{\prime} d t$. Formula (4.1) proves that

$$
\Xi^{m}[T]\left(A_{L}^{m}\right)=\mathrm{Op}\left(a_{T}^{\prime}\right),
$$

where, as in $\oint 3.1$, we call $A_{L}^{m}:=\operatorname{Op}\left(a^{\prime}\right)$.

The existence of $\Xi^{m}[\infty]\left(A_{L}^{m}\right)$ is a trivial consequence of the Heisenberg evolution: we can easily figure it out looking at its matrix elements w.r.t. the bases $\left\{e_{\alpha}^{(k)}\right\} \subset L_{(k)}^{2}\left(T_{L}^{m}\right)$. These bases diagonalize the Hamiltonian $H_{m}$, as well as any operator function of $P$ only. We call such eigenvalues

$$
E_{\alpha}^{(k)}=\frac{1}{2}(\alpha+k)^{2}=\frac{1}{2} \sum_{i=1}^{n}\left(\alpha_{i}+k_{i}\right)^{2}
$$

Now it is easy to see that, $\forall k \in[0,1 / L)^{m}, \alpha, \gamma \in\left(T_{L}^{m}\right)^{*}$,

$$
\left\langle e_{\alpha}^{(k)}, \Xi^{m}[\infty]\left(A_{L}^{m}\right) e_{\gamma}^{(k)}\right\rangle=\left\langle e_{\alpha}^{(k)}, A_{L}^{m} e_{\gamma}^{(k)}\right\rangle \delta_{E_{\alpha}^{(k)}, E_{\gamma}^{(k)}}
$$

where $\delta$ is the Kronecker $\delta$-function. This formula shows that $\Xi^{m}[\infty]\left(A_{L}^{m}\right)$ is well defined on all vectors in $D\left(A_{L}^{m}\right)$.

One might think to prove now the statement regarding $J(\infty, L, m)$ by simply substituting the Heisenberg invariant operator $\Xi^{m}[\infty]\left(A_{L}^{m}\right)$ to $\mathrm{Op}\left(a^{\prime}\right)$ and $\operatorname{Op}\left(b^{\prime}\right)$ in Theorem 3.2. We cannot quite do this, since such operator is not in general pseudo-differential. It is obvious, though, that it can be approximated to any extent by pseudo-differential operators, and the result would follow by density. However, as remarked in $\$ 3.1$, we have not defined a proper $C^{*}$-algebra for the infinite-particle system. Thus, we cannot talk of any density and have to prove the theorem directly. 
$\Xi^{m}[\infty]\left(A_{L}^{m}\right)$, roughly speaking, represents the quantization of $a_{\infty}^{\prime}:=\lim _{T \rightarrow \infty} a_{T}^{\prime}$, which is not in general a symbol, being possibly not even continuous. But simple considerations based upon the trivial dynamics over $\Lambda_{L}^{m}$ (see also the remark after the statement of this theorem) show that it is almost everywhere (namely for $p=\left(p_{1}, \ldots, p_{m}\right)$ having rationally independent components) equal to

$$
c^{\prime}(p, q)=c^{\prime}(p):=\frac{1}{L^{m}} \int_{T_{L}^{m}} a^{\prime}(p, q) d q
$$

which is a symbol. Moreover we denote it $c^{\prime}$ since one can straightforwardly find a $c \in \mathcal{A}$ such that $c^{\prime}=c \circ \prod_{L}^{m}$. The whole idea of this proof is exactly to show that, in some sense, $\operatorname{Op}\left(c^{\prime}\right)$ is a.e. equal to $\Xi^{m}[\infty]\left(A_{L}^{m}\right)$, so that the former can be substituted to the latter in the definition of $J(\infty, L, m)$ in order to apply Theorem 3.2 (also compare (3.19) and (3.18)).

We first remark some basic properties of $\mathrm{Op}\left(c^{\prime}\right)$. Since $c^{\prime}(p, q)=c^{\prime}(p)$ then $\mathrm{Op}\left(c^{\prime}\right)$ is diagonal w.r.t. $\left\{e_{\alpha}^{(k)}\right\}$. Its diagonal matrix elements, using (3.14), are found to be

$$
\left\langle e_{\alpha}^{(k)}, \operatorname{Op}\left(c^{\prime}\right) e_{\alpha}^{(k)}\right\rangle=\frac{c^{\prime}(\alpha+k)}{L^{m}}=\frac{1}{L^{m}} \int_{T_{L}^{m}} a^{\prime}(\alpha+k, q) d q=\left\langle e_{\alpha}^{(k)}, A_{L}^{m} e_{\alpha}^{(k)}\right\rangle,
$$

showing incidentally, as it ought to be, that $\mathrm{Op}\left(c^{\prime}\right)$ is invariant for time evolution. More importantly, (1.24), together with (4.22), implies that $\mathrm{Op}\left(c^{\prime}\right)^{(k)}=$ $\left(\Xi^{m}[\infty]\left(A_{L}^{m}\right)\right)^{(k)} \Xi^{\mathbb{3}}$ for those $k \in[0,1 / L)^{m}$ for which $H_{m}^{(k)}$ is diagonal.

Using (2.19) over a generic $f_{\lambda}$ picked up from the set of states satisfying (3.2), one can see that $w_{\lambda}(p, q)$ contains a (possibly countable) sum of $\delta$ functions in $p$. This simple argument shows that, in order for $\left\{f_{\lambda}\right\}$ to verify (3.2), a factor of the measure space $\left(X_{L}^{m}, d \theta\right)$ must be $\left([0,1 / L)^{m}, d \tau(k)\right)$, with $d \tau$ absolutely continuous w.r.t. the Lebesgue measure. [4 So if we prove that $\sigma\left(H_{m}^{(k)}\right)$ is simple for Lebesgue-almost all $k$ 's, then

$$
J(\infty, L, m):=\int_{X_{L}^{m}}\left\|\left(\mathrm{Op}\left(c^{\prime}\right)-P_{\rho, 2 \beta}(a)\right) g_{\lambda}\right\|^{2} d \nu(\lambda)
$$

\footnotetext{
${ }^{13}$ Remember that with $A^{(k)}$ we denote $A_{\mid L_{(k)}^{2}\left(T_{L}^{m}\right)}$ as explained in $\$ 2.2$.

${ }^{14}$ This is a manifestation of the fact that all fibers $L_{(k)}^{2}\left(T_{L}^{m}\right)$ need to be taken into account, as mentioned in $\$ 2.2$, Remark 1 . We can convince ourselves of this also looking at the two examples of $\left\{f_{\lambda}\right\}$ we have explicitly written: the Fourier basis in $\$ 3.1$ and the coherent states in the appendix, $\$$ A.1. In both cases $\left.\left(X_{L}^{m}, d \theta\right)=\left([0,1 / L)^{m}, d k\right)\right) \times$ some measure.
} 
and we can apply Theorem 3.2 with $a=b=c-P_{\rho, 2 \beta}(a)$, which is time invariant. This would complete the proof of the first claim.

Rescaling (4.21) by a factor $L^{m}$, what we need is equivalent to the following

\section{Lemma 4.4}

$$
\mid\left\{k \in[0,1)^{m} \mid \exists j, n \in \mathbb{Z}^{m} \text { s.t. }(j+k)^{2}=(n+k)^{2}\right\} \mid=0 .
$$

Proof of Lemma 4.4. 四 Thinking of it as a geometric problem in $\mathbb{R}^{m}$, when such $j, n$ exist, then $-k$ lies in the axial hyperplane of the segment joining $j$ to $n$, i.e. the set of points in the space equally distant from $j$ and $n$. By very construction there is only a countable number of such hyperplanes. Q.E.D.

As far as the last statement of Theorem 3.3 is concerned, we see again that it cannot be derived as a corollary of the mixing theorem since we are taking time limits of both operators. But we can give a direct proof using the techniques of $\$ 4.1$ and the classical ergodicity result contained in Theorem 2.2 .

Exactly as in (4.2) and (4.3) we can write

$$
J(T, L, m)=\int_{\Lambda_{L}^{m}}\left[\left(a_{T}^{\prime}-P_{\rho, 2 \beta}(a)\right) \sharp\left(a_{T}^{\prime}-P_{\rho, 2 \beta}(a)\right)\right](p, q)\left[e^{-2 \beta \mathcal{H}_{m}(p)} d p d q\right]_{N},
$$

having used (4.20). We have become familiar with this object in $\$ 4.1$, and we have seen that integrating - w.r.t. the Gibbs measure - the Weyl composition of two functions means integrating the product of the two functions, one of which scrambled by a convolution (see (4.3), (4.4) and (4.14)). At the thermodynamic limit, this amounts to say that

$$
\lim _{\substack{m, L \rightarrow \infty \\ m / L \rightarrow \rho}} J(T, L, m)=P_{\rho, 2 \beta}\left(\left(a_{T}-P_{\rho, 2 \beta}(a)\right) c^{(T)}\right),
$$

where $c^{(T)}$ is the limit of the "scrambled functions" constructed upon $\left(a_{T}-\right.$ $\left.P_{\rho, 2 \beta}(a)\right)$. Its existence is granted by Lemmas 4.3 and 4.2, which yielded

\footnotetext{
${ }^{15}$ I thank D.Dolgopyat for this simple proof.
} 
(4.17). From the construction we have just recalled it can be seen that if $\left(a_{T}-P_{\rho, 2 \beta}(a)\right)$ is bounded then $c^{(T)}$ is as well.

A remark is in order here: in $\$ 4.1$ we have worked with symbols belonging to $\mathcal{B}^{(n)}$, and those are unbounded by definition, being the limits of functions like $N_{L, m}^{(n)}(\beta)$ defined in (4.8). But a simple argument shows that a bounded $a \in \mathcal{A}=\sigma\left(\oplus \mathcal{B}^{(n)}\right)$ remains bounded after the above procedure, since, roughly speaking, it gets deformed in the same way in each of its $\mathcal{B}^{(n)}$-components.

Finally, we can apply Lebesgue dominated convergence in (4.27) since the integrand function is bounded and tends pointwise to zero as $T \rightarrow \infty$. Thus, the $T$-limit of (4.27) gives the last statement in Theorem 3.3, whence the end of the proof.

Q.E.D.

\section{Acknowledgments}

I would like to thank S.Graffi and Ya.G.Sinai for addressing this problem to me, giving stimulation as well as useful advices. I also wish to thank A.Parmeggiani and A.Martinez for interesting discussions on this subject. A grant from I.N.F.N. is highly acknowledged.

\section{A Appendix}

\section{A.1 Coherent states for the cylinder.}

We begin this section by recalling some notions about the Bloch decomposition (2.9), following $[\mathbb{R}-\mathrm{S}]$ and $[\mathrm{dB}-\mathrm{G}]$. The idea is very simple: given a function $f \in L^{2}\left(\mathbb{R}^{m}\right)$, and therefore its Fourier transform $\widehat{f}(p)$, we pick up from the latter only the terms at $p=\xi+k,\left(\xi \in\left(T_{L}^{m}\right)^{*}\right)$ to construct $f^{(k)}$ which clearly lies in $L_{(k)}^{2}\left(T_{L}^{m}\right)$.

In formula

$$
f^{(k)}(x):=\frac{1}{L^{m}} \sum_{\xi \in\left(T_{L}^{m}\right)^{*}} \widehat{f}(\xi+k) e^{2 \pi i(\xi+k) \cdot x} .
$$

Considering the scalar products in the dual spaces (respectively $L^{2}\left(\mathbb{R}^{m}\right)$ and $\left.\ell^{2}\left((\mathbb{Z} / L)^{m}+k\right)\right)$, it is easy to see the decomposition property which justifies 
(2.9):

$$
\langle f, g\rangle_{L^{2}\left(\mathbb{R}^{m}\right)}=\int_{[0,1 / L)^{m}}\left\langle f^{(k)}, g^{(k)}\right\rangle_{L_{(k)}^{2}\left(T_{L}^{m}\right)} d k
$$

An explicit formula for $f^{(k)}$, more direct than (A.1), is also computable with the aid of the Poisson summation formula:

$$
f^{(k)}(x)=\sum_{n \in \mathbb{Z}^{m}} e^{-2 \pi i L n \cdot k} f(x+L n)
$$

We can now proceed to the construction of a remarkable example of states satisfying the assumptions of the theorems. Let a family of generalized coherent states for the Euclidean $2 m$-dimensional phase space be given

$$
f_{(u, v)}:=T(-u, v) f_{0}
$$

as constructed in $[\mathbb{P}]$, where $(u, v) \in \mathbb{R}^{2 m}$ and $f_{0} \in L^{2}\left(\mathbb{R}^{m}\right)$, usually a Gaussian centered at the origin. According to our preparatory remark, we give the following

Definition A.1 dB-G The family $f_{(u, v)}^{(k)}$ where $(u, v, k) \in X_{L}^{m}:=T_{L}^{m} \times \mathbb{R}^{m} \times$ $[0,1 / L)^{m}$ constructed as above is called a set of coherent states on $\Lambda_{L}^{m}$.

We endow $X_{L}^{m}$ with the measure $d \theta(u, v, k):=d u d v d k$ and check that they verify the hypothesis of the theorem.

We shall work on the Fourier antitransform of $w_{\lambda}$, i.e. on the FourierWigner function relative to the state $f_{\lambda}$.

$$
\begin{aligned}
& \int_{\Lambda_{L}^{m}} d u d v \int_{[0,1 / L)^{m}} d k\left\langle f_{(u, v)}^{(k)}, T^{(k)}(\eta, \xi) f_{(u, v)}^{(k)}\right\rangle_{L_{(k)}^{2}\left(T_{L}^{m}\right)}= \\
= & \int_{\Lambda_{L}^{m}} d u d v\left\langle f_{(u, v)}, T(\eta, \xi) f_{(u, v)}\right\rangle_{L^{2}\left(\mathbb{R}^{m}\right)}= \\
= & \int_{\Lambda_{L}^{m}} d u d v\left\langle T(-u, v) f_{0}, T(-u, v) T(\eta, \xi) f_{0}\right\rangle_{L^{2}\left(\mathbb{R}^{m}\right)} e^{2 \pi i(\eta \cdot v+\xi \cdot u)}=(\mathrm{A} .5) \\
= & \int_{\Lambda_{L}^{m}} d u d v\left\langle f_{0}, T(\eta, \xi) f_{0}\right\rangle_{L^{2}\left(\mathbb{R}^{m}\right)} e^{2 \pi i(\eta \cdot v+\xi \cdot u)}= \\
= & \left\langle f_{0}, T(\eta, \xi) f_{0}\right\rangle_{L^{2}\left(\mathbb{R}^{m}\right)} \delta_{\xi} \delta(\eta)=\delta_{\xi} \delta(\eta),
\end{aligned}
$$

which is another way to state (3.2). The first step is justified by (A.2) and the third by the commutation relations in the Heisenberg group. 
Finally, this set of coherent states is perhaps the most important among the possible collections one could choose. As a matter of fact, such states are indeed introduced to be as localized as the Heisenberg principle permits, as clearly explained in $\mathrm{dB}-\mathrm{G}$. Had we performed a limit $\hbar \rightarrow 0$, exploiting the Wigner function as a measure of the degree of localization of a state, we would have seen that

$$
W_{f_{(u, v)}^{(k)}, f_{(u, v)}^{(k)}}(p, q) \longrightarrow \delta(p-u) \delta(q-v) \quad \text { as } \hbar \rightarrow 0
$$

where the dependence on $\hbar$ is implicit in the construction of $f_{(u, v)}^{(k)}$. This is why one can say that such a state is a good analogue of a point in the phase space. Therefore we see that the physical meaning of our quantum ergodic properties gets clearer and more classical, of course, in the semiclassical regime.

\section{A.2 Proof of Lemma 4.1.}

Let us check that equality on all the matrix elements with respect to the standard basis of $L_{(k)}^{2}\left(T_{L}^{m}\right),\left\{e_{\alpha}^{(k)}\right\}_{\alpha \in\left(T_{L}^{m}\right)^{*}}$ introduced in $\$ 2.2$. It is easily computed that

$$
T^{(k)}(\eta, \xi) e_{\alpha}^{(k)}=e^{2 \pi i \eta \cdot(\xi / 2+\alpha+k)} e_{\alpha+\xi}^{(k)} .
$$

If we now denote $c^{t}(p, q):=\left(c \circ \phi_{L}^{m}[t]\right)=c(p, q+p t)$, we can compute its Fourier transform which turns out to be $\widehat{c^{t}}(\eta, \xi)=\widehat{c}(\eta-\xi t, \xi)$. Thus, substituting into (2.14) and changing variable,

$$
\mathrm{Op}\left(c^{t}\right):=\frac{1}{L^{m}} \sum_{\xi \in\left(T_{L}^{m}\right)^{*}} \int_{\mathbb{R}^{m}} \widehat{c}(\eta, \xi) T(\eta+\xi t, \xi) d \eta .
$$

In order for the statement to hold for every $c$, it is a necessary and sufficient condition that $\forall \alpha, \gamma \in\left(T_{L}^{m}\right)^{*}$

$$
\left\langle e_{\alpha}^{(k)}, T(\eta+\xi t, \xi) e_{\gamma}^{(k)}\right\rangle=\left\langle e_{\alpha}^{(k)}, e^{2 \pi i t H_{m}} T(\eta, \xi) e^{-2 \pi i t H_{m}} e_{\gamma}^{(k)}\right\rangle
$$

Using (A.7) we find on the r.h.s.

$$
\left\langle e_{\alpha}^{(k)}, T(\eta+\xi t, \xi) e_{\gamma}^{(k)}\right\rangle=e^{2 \pi i(\eta+\xi t) \cdot(\xi / 2+\gamma+k)} \delta_{\alpha, \gamma+\xi}
$$


Since $P^{(k)} e_{\alpha}^{(k)}=(\alpha+k) e_{\alpha}^{(k)}$, on the l.h.s. we have

$$
\begin{aligned}
& \left\langle e_{\alpha}^{(k)}, e^{2 \pi i t H_{m}} T(\eta, \xi) e^{-2 \pi i t H_{m}} e_{\gamma}^{(k)}\right\rangle= \\
= & e^{\pi i(\alpha+k)^{2} t} e^{-\pi i(\gamma+k)^{2} t} e^{2 \pi i \eta \cdot(\xi / 2+\gamma+k)} \delta_{\alpha, \gamma+\xi} \\
= & e^{2 \pi i((\xi / 2+\gamma+k) \xi t+(\xi / 2+\gamma+k) \eta)} \delta_{\alpha, \gamma+\xi},
\end{aligned}
$$

where we have substituted for $\alpha$ its value $\gamma+\xi$. This relation finally verifies (A.9).

Q.E.D.

\section{A.3 Convolutions over tori and over Euclidean spaces.}

The purpose of this section is to clarify the meaning of the symbol $*_{L}$, indicating $q$-convolution over $T_{L}^{m}$, when applied to functions that are in principle defined over larger sets, like the functions $b \circ \Pi_{L}^{m}$, for instance. Also, we want to understand how this is related to $*_{\infty}$, the ordinary convolution on $\mathbb{R}^{m}$, when $L \rightarrow \infty$.

Since the arguments here are essentially descriptive, we specialize to one dimension, without loss of generality. If $f$ is a nice function defined on $\mathbb{R}$, denote by $f^{(L)}$, its periodic restriction, i.e. the function, defined again on $\mathbb{R}$, which is $L$-periodic and coincides with $f$ on $[-L / 2, L / 2)$. Then if $g$ is also a nice function on $\mathbb{R}$, we define

$$
\begin{aligned}
\left(f *_{L} g\right)(x) & :=\left(f^{(L)} * g^{(L)}\right)(x)=\int_{-L / 2}^{L / 2} f^{(L)}(y) g^{(L)}(x-y) d y= \\
& =\int_{-L / 2}^{L / 2} f(y) g^{(L)}(x-y) d y .
\end{aligned}
$$

Thus, for instance, $\left(f *_{L} g\right)(x) \neq \int_{-L / 2}^{L / 2} f(y) g(x-y) d y$. As $L \rightarrow \infty$, however, we expect this to be approximately true, at least for a fixed $x \in \mathbb{R}$. As a matter of fact, requiring some properties of $f$ and $g$, one can prove a useful lemma. Call $S_{R}:=[-R, R]$.

Lemma A.2 Suppose $f \in C_{0}^{\infty}(\mathbb{R})$ and supp $f \subseteq S_{R}$. Assume also that $|g|$ vanishes monotonically at infinity. Defining

$$
h(x)=\left(f *_{L} g-f *_{\infty} g\right)(x),
$$


Ergodic Properties of the Quantum Ideal Gas ...

then one has, for L sufficiently large,

$$
h(x) \begin{cases}\leq M(|g(-L / 2)|+|g(L / 2-R)|) & \text { for } x \in[-L / 2,-L / 2+R) \\ =0 & \text { for } x \in[-L / 2+R, L / 2-R] \\ \leq M(|g(L / 2)|+|g(-L / 2+R)|) & \text { for } x \in(L / 2-R, L / 2]\end{cases}
$$

where $M:=R \max |f|$.

Proof of Lemma A.2. Take $L$ so large that $L / 2>R$ and $|g|$ is increasing in $(-\infty,-L / 2+R]$ and decreasing in $[L / 2-R,+\infty)$.

Looking at (A.12) and recalling the hypothesis on $f$, we can write

$$
\left(f *_{L} g\right)(x)=\int_{S_{R}} f(y) g^{(L)}(x-y) d y .
$$

Hence

$$
h(x)=\int_{S_{R}} f(y)\left(g^{(L)}-g\right)(x-y) d y .
$$

Now, $g^{(L)}(x-y)$ coincides with $g^{(L)}(x-y)$ when $x-y \in S_{L / 2}$, that is, when $y \in[x-L / 2, x+L / 2]$. So (A.14) is rewritten as

$$
h(x)=\int_{S_{R} \backslash\left(x+S_{L / 2}\right)} f(y)\left(g^{(L)}-g\right)(x-y) d y .
$$

It is easily seen that if $x \in[-L / 2+R, L / 2-R]$, then $S_{R} \subseteq\left(x+S_{L / 2}\right)$ such that $h(x)=0$ and part of the claim is proved. If $x \in[-L / 2,-L / 2+R)$, making the change of variable $z=x-y$, (A.14) gives

$$
\begin{aligned}
|h(x)| & =\left|\int_{x-R}^{-L / 2} f(x-z)\left(g^{(L)}-g\right)(z) d z\right| \leq \\
& \leq \max |f| \int_{-L / 2-R}^{-L / 2}\left(\left|g^{(L)}(z)\right|+|g(z)|\right) d z .
\end{aligned}
$$

Since by definition, for $z<-L / 2, g^{(L)}(z)=g(z+L)$, the monotonicity property of $g$ gives the first case in the statement of the lemma. The third case is of course analogous.

Q.E.D. 


\section{A.4 Proof of Lemma 4.3.}

Before even getting started, let us agree upon denoting, throughout this proof, by $(p, q)$ all momentum-coordinate variables, be they defined on $\Lambda_{L}^{m}$ or on $\mathbb{R}^{2 n}$ or on $\Lambda_{L}^{1}$. Notice that, in the proof of Theorem 3.2, we referred to $n$-dimensional variables as $\left(p^{\prime}, q^{\prime}\right)$.

The whole idea here is to realize that, if $n$ is fixed, the function $\Phi_{L}^{n}$ defined as in (4.5), gets closer and closer, in $\Lambda_{L}^{n}$, to

$$
\begin{aligned}
\Phi_{\infty}^{n}(p, q) & =e^{\beta p^{2}} \int_{\mathbb{R}^{n}} d \xi e^{-\beta(p-\xi / 2)^{2}} e^{2 \pi i \xi \cdot q}= \\
& =e^{\beta p^{2}} e^{4 \pi i p \cdot q}\left(\frac{4 \pi}{\beta}\right)^{n / 2} e^{-\left(4 \pi^{2} / \beta\right) q^{2}}= \\
& =e^{\beta p^{2}} e^{4 \pi i p \cdot q} v^{n}(q),
\end{aligned}
$$

where $v(q):=\sqrt{4 \pi / \beta} e^{-\left(4 \pi^{2} / \beta\right) q^{2}}$. In the following we will use repeatedly the asymptotic estimate $v(L / 2)=\mathcal{O}\left(L^{-\infty}\right)$.

Anyway, $\Phi_{\infty}^{n}$ is defined by the fact that it has the same Fourier spectrum of $\Phi_{L}^{n}$, suitably extended to all of $\mathbb{R}^{n}$. If we denote by $\sim$ the $q$-Fourier transform over $T_{L}^{n}$, then this amounts to say that, for $\xi \in\left(T_{L}^{n}\right)^{*}=(\mathbb{Z} / L)^{n}$,

$$
\int_{\mathbb{R}^{n}} \Phi_{\infty}^{n}(p, q) e^{-2 \pi i \xi \cdot q} d q=e^{\beta p^{2}} e^{-\beta(p-\xi / 2)^{2}}=: \widetilde{\Phi}_{L}^{n}(p, \xi) .
$$

So the best candidate for $\gamma_{\infty}$ is $\beta *_{\infty} \Phi_{\infty}^{n}$. The symbol $*_{\infty}$ designates the $q$-convolution over $\mathbb{R}^{n}$, as explained in $\S$ A.3. First of all, such a $\gamma_{\infty}$ verifies (4.16): this is a consequence of the fact that $\int_{\mathbb{R}^{n}} \Phi_{\infty}^{n}=1$, which is easily verified. Let us proceed to the proof of (4.15).

Recalling the warning in $\$$ A.3, the main inequality will be

$$
\begin{gathered}
\left\|\gamma_{L}-\gamma_{\infty}\right\|_{L^{2}\left(\Lambda_{L}^{n}, \mu_{L}^{n}\right)}=\left\|\beta *_{L} \Phi_{L}^{n}-\beta *_{\infty} \Phi_{\infty}^{n}\right\|_{L^{2}\left(\Lambda_{L}^{n}, \mu_{L}^{n}\right)} \leq \\
\leq\left\|\beta *_{L}\left(\Phi_{L}^{n}-\Phi_{\infty}^{n}\right)\right\|_{L^{2}\left(\Lambda_{L}^{n}, \mu_{L}^{n}\right)}+\left\|\beta *_{L} \Phi_{\infty}^{n}-\beta *_{\infty} \Phi_{\infty}^{n}\right\|_{L^{2}\left(\Lambda_{L}^{n}, \mu_{L}^{n}\right)}
\end{gathered}
$$

The leftmost term can be worked out pointwise, using (A.17) and Lemma A.2 of $\$$ A.3. If $(p, q) \in \Lambda_{L}^{n}$,

$$
\left|\left(\beta *_{L} \Phi_{\infty}^{n}-\beta *_{\infty} \Phi_{\infty}^{n}\right)(p, q)\right| \leq M v^{n}\left(\frac{L}{2}-R\right),
$$


where $M \simeq \max \left(|\beta(p, q)| e^{\beta p^{2}}\right)$ and $R$ is the radius of the ball containing $\operatorname{supp} \beta$. Now, since $\mu_{L}^{n}$ is a probability measure,

$$
\left\|\beta *_{L} \Phi_{\infty}-\beta *_{\infty} \Phi_{\infty}\right\|_{L^{2}\left(\Lambda_{L}^{n}, \mu_{L}^{n}\right)}=\mathcal{O}\left(L^{-\infty}\right) .
$$

To work out the other term in (A.19) we employ the ideas stated at the beginning of this section about $\Phi_{\infty}^{n}$. We start by applying the well-known convolution inequality to our case. We have

$$
\int_{T_{L}^{n}}\left|\beta *_{L}\left(\Phi_{L}^{n}-\Phi_{\infty}^{n}\right)(p, q)\right|^{2} d q \leq\|\beta(p, \cdot)\|_{L^{1}\left(T_{L}^{n}, d q\right)}^{2}\left\|\left(\Phi_{L}^{n}-\Phi_{\infty}^{n}\right)(p, \cdot)\right\|_{L^{2}\left(T_{L}^{n}, d q\right)}^{2} .
$$

Notice that $\|\beta(p, \cdot)\|_{L^{1}\left(T_{L}^{n}\right)}=\|\beta(p, \cdot)\|_{L^{1}\left(\mathbb{R}^{n}\right)}$, since $\beta$ is compactly supported. We also see that

$$
\left\|\left(\Phi_{L}^{n}-\Phi_{\infty}^{n}\right)\left(p_{1}, \ldots, p_{n}, \cdot\right)\right\|_{L^{2}\left(T_{L}^{n}, d q\right)}^{2}=\prod_{i=1}^{n}\left\|\left(\Phi_{L}-\Phi_{\infty}\right)\left(p_{i}, \cdot\right)\right\|_{L^{2}\left(T_{L}, d q_{i}\right)}^{2},
$$

since $\Phi_{L}^{n}$ and $\Phi_{\infty}^{n}$ are completely factorizable: we call, obviously, $\Phi_{L}$ and $\Phi_{\infty}$ their one-dimensional versions, on which we are immediately going to work. As anticipated at the beginning of this section, we will be a little imprecise and use again the label $(p, q)$ for $\left(p_{i}, q_{i}\right)$. Now

$$
\left\|\left(\Phi_{L}-\Phi_{\infty}\right)(p, \cdot)\right\|_{L^{2}\left(T_{L}\right)}^{2}=\frac{1}{L} \sum_{\xi \in(\mathbb{Z} / L)}\left|\widetilde{\Phi}_{L}(p, \xi)-\widetilde{\Phi}_{\infty}(p, \xi)\right|^{2},
$$

with $\widetilde{\Phi}_{\infty}(p, \xi)=\int_{-L / 2}^{L / 2} \Phi_{\infty}(p, q) e^{-2 \pi i \xi q} d q$. Looking back at (A.18) and using definition (A.17), we can write

$$
\begin{aligned}
& \left|\widetilde{\Phi}_{L}(p, \xi)-\widetilde{\Phi}_{\infty}(p, \xi)\right|= \\
= & \left|\int_{\mathbb{R} \backslash T_{L}} \Phi_{\infty}(p, q) e^{-2 \pi i \xi q} d q\right|= \\
= & 2 e^{\beta p^{2}}\left|\int_{L / 2}^{+\infty} v(q) \cos (2 \pi(\xi-2 p) q) d q\right|= \\
= & 2 e^{\beta p^{2}}\left|\left[\frac{v(q) \sin (2 \pi(\xi-2 p) q)}{2 \pi(\xi-2 p)}\right]_{L / 2}^{+\infty}-\int_{L / 2}^{+\infty} \frac{v^{\prime}(q) \sin (2 \pi(\xi-2 p) q)}{2 \pi(\xi-2 p)} d q\right| \leq \\
\leq & e^{\beta p^{2}} g(\xi-2 p) v(L / 2),
\end{aligned}
$$


where $g(x)$ is a continuous function defined on $\mathbb{R}$ behaving like $|x|^{-1}$ for large values of $x$. Note that, as $L \rightarrow \infty,(1 / L) \sum_{\xi \in(\mathbb{Z} / L)} g^{2}(\xi-2 p) \rightarrow \int_{\mathbb{R}} g^{2}(\xi) d \xi=$ : $K$, uniformly for $p$ in a compact set. So, looking at (A.22), and merging up (A.23), (A.24) and (A.25), we have

$$
\begin{aligned}
& \left\|\beta *_{L}\left(\Phi_{L}^{n}-\Phi_{\infty}^{n}\right)\right\|_{L^{2}\left(\Lambda_{L}^{n}, \mu_{L}^{n}\right)}^{2}= \\
= & \frac{1}{L^{n}} \int_{\mathbb{R}^{n}} \| \beta *_{L}\left(\Phi_{L}^{n}-\Phi_{\infty}^{n}(p, \cdot) \|_{L^{2}\left(T_{L}^{n}, d q\right)}^{2} d \breve{\mu}^{n}(p) \leq\right. \\
\leq & \left(\frac{(K+1) v^{2}(L / 2)}{L^{n}}\right)^{n} \int_{\mathbb{R}^{n}}\|\beta(p, \cdot)\|_{L^{1}\left(\mathbb{R}^{n}\right)}^{2} e^{2 \beta p^{2}} d \breve{\mu}^{n}(p)=\mathcal{O}\left(L^{-\infty}\right),
\end{aligned}
$$

since $\beta$ has compact support. Inserting (A.21) and (A.26) into the fundamental inequality (A.19) the proof is completed.

Q.E.D.

\section{References}

[B] F.BenatTi Deterministic Chaos in Infinite Quantum System Springer-Verlag, Berlin, New York, 1993

[B-R] O.Bratteli, D.W.RoBinson Operator Algebras and Quantum Statistical Mechanics, Vol. I and II Text and Monographs in Physics, Springer-Verlag, 1987

[dB-G] S.DE Bièvre, J.A.GonzÁLes Semiclassical Behaviour of Coherent States on the Circle in: Proc. of 11th workshop on geometric methods in physics, Bialowieza, Poland, World Scientific, 1993. S.T.Ali, I.M.Mladenov, A.Odzijewicz eds.

[Fo] G.Folland Harmonic Analysis in Phase Space Princeton Univ. Press, Princeton, 1988

[JL-P] G.Jona-Lasinio, C.Presilla Chaotic Properties of Quantum Many Body Systems in the Thermodynamic Limit currently at cond-mat@xxx.lanl.gov/9601056

[JL-P-C] G.Jona-Lasinio, C.Presilla, F.CAPAsso Chaotic Quantum Phenomena without Classical Counterpart Phys. Rev. Lett. 68 (1992), 2269-2273 
Ergodic Properties of the Quantum Ideal Gas ...

[G-L-A] S.Goldstein, J.L.Lebowitz, M.Aizenman Ergodic properties of infinite systems Proc. of 1973 Battelle Rencontre, J.Moser ed., Lectures Notes in Physics 38, (1974), 144-177

[G-M] S.Graffi, A.Martinez Ergodic Properties of Infinite Harmonic Crystals: An Analytic Approach currently at chaodyn@xyz.lanl.gov/9602003

[P] A.M.Perelomov Generalized Coherent States and Their Applications Texts and Monographs in Physics, Springer-Verlag, 1986

[R-S] M.ReED, B.Simon Methods of Modern Mathematical Physics IV: Analysis of Operators Academic Press, 1978

[R] D.Ruelle Statistical Mechanics: Rigorous Results W.A.Benjamin, New York, 1969

[Sh] A.M.Shubin Pseudodifferential Operators and Spectral Theory Springer-Verlag, 1987.

[S] Ya.G.Sinai Introduction to Ergodic Theory Princeton Univ. Press, Princeton, 1976.

[V-S] K.L.Volkovyski, Ya.G.Sinai Ergodic Properties of an Ideal Gas with Infinitely Many Degrees of Freedom Funct. Anal. Appl. 5, (1971), 185-187.

[VN] J.Von Neumann Beweis des Ergodensatzes und des H-Theorems in der Neuen Mechanik Zschr.f.Physik 57, (1929), 30-70. 